\title{
Dynamic Analysis of a Phytoplankton-Fish Model with Biological and Artificial Control
}

\author{
Yapei Wang, ${ }^{1,2}$ Min Zhao,, ${ }^{2,3}$ Xinhong Pan, ${ }^{1,2}$ and Chuanjun Dai ${ }^{2,3}$ \\ ${ }^{1}$ School of Mathematics and Information Science, Wenzhou University, Wenzhou, Zhejiang 325035, China \\ ${ }^{2}$ Zhejiang Provincial Key Laboratory for Water Environment and Marine Biological Resources Protection, \\ Wenzhou University, Wenzhou, Zhejiang 325035, China \\ ${ }^{3}$ School of Life and Environmental Science, Wenzhou University, Wenzhou, Zhejiang 325035, China
}

Correspondence should be addressed to Min Zhao; zmcn@tom.com

Received 24 October 2013; Accepted 17 January 2014; Published 17 March 2014

Academic Editor: Zhan Zhou

Copyright (C) 2014 Yapei Wang et al. This is an open access article distributed under the Creative Commons Attribution License, which permits unrestricted use, distribution, and reproduction in any medium, provided the original work is properly cited.

We investigate a nonlinear model of the interaction between phytoplankton and fish, which uses a pair of semicontinuous systems with biological and artificial control. First, the existence of an order-1 periodic solution to the system is analyzed using a Poincaré map and a geometric method. The stability conditions of the order-1 periodic solution are obtained by a theoretical mathematical analysis. Furthermore, based on previous analysis, we investigate the bifurcation in the order-1 periodic solution and prove that the order-1 periodic solution breaks up an order-1 periodic solution at least. In addition, the transcritical bifurcation of the system is described. Finally, we provide a series of numerical results that illustrate the feasibility of the theoretical results. Based on the theoretical and numerical results, we analyzed the feasibility of biological and artificial control, which showed that biological and artificial methods can control phytoplankton blooms. These results are expected to be useful for the study of phytoplankton dynamics in aquatic ecosystems.

\section{Introduction}

Phytoplankton plays an important role in ecology and the climate because it participates in the global carbon cycle as the base of the food chain [1]. A feature of plankton populations is the occurrence of rapid population explosions and almost equally rapid declines, which are separated by periods of almost stationary high or low population levels [2]. This phenomenon is known as a "bloom." In specific environmental conditions, lakes, reservoir, and marine waters may experience plankton or algae blooms [3, 4]. For example, eutrophication may cause blue-green algae, which are very small plankton species with rapid rates of reproduction, to bloom frequently in the Zeya Reservoir in Wenzhou, which is located in a subtropical region. This may degrade the water quality and could deprive millions of people of drinking water. In particular, some types of phytoplankton are rich in neurotoxins, which can cause substantial mortality in fish, while the toxins absorbed by shellfish may cause paralysis and death in sea birds and humans [5]. Thus, it is very important to control the growth of phytoplankton.
In general, physical methods (e.g., artificial refloatation and removal using machines), chemical methods (e.g., adding chemical reagents to the water), and biological methods (e.g., releasing natural enemies or competitors) are used to kill phytoplankton or restrain their growth when blooms occur. However, physical methods are rarely effective in preventing phytoplankton blooms, while chemical methods may cause secondary pollution. Furthermore, the phytoplankton continues to reproduce when the concentration of chemical reagents in the water drops to certain levels. In particular, when the chemical reagents beyond a certain level, the algae removal rate may reach $100 \%$ within $24 \mathrm{~h}$ [6]. It may be possible to break the ecological balance but this is not the goal. In the present study, physical and chemical methods are referred to as artificial methods.

In the food chain, phytoplankton is not the top trophic level, and some higher trophic levels, such as filter-feeding fish, capture and feed upon phytoplankton. Thus, biological methods can be used to control the growth of phytoplankton in an effective manner. Liu and Xie [7] conducted in situ enclosure experiments in a lake over the course of three 
years and showed that intense stocking with filter-feeding fishes, that is, silver carp (Hypophthalmichthys molitrix) and bighead carp (Aristichthys nobilis), played a decisive role in the elimination of blue algae blooms from the lake. The results indicated that silver carp and bighead carp controlled the blue algae blooms effectively, and the effective biomass required to contain the blooms was determined to be $46-50 \mathrm{~g} / \mathrm{m}^{3}$. In another study [8], a mesocosm experiment was conducted to assess the impact of a moderate silver carp biomass $\left(41 \mathrm{~g} / \mathrm{m}^{3}\right.$ or $850 \mathrm{~kg} / \mathrm{ha}$ ) on the plankton community and the water quality of the eutrophic Paranoá Reservoir (Brasília, Brazil). The results, as well as other successful examples of blue-green control using silver carp in lakes and reservoirs [9-11], suggest that the use of silver carp is a promising management tool for suppressing excess filamentous blue-green algae, such as that in Paranoá Reservoir, and for controlling Microcystis blooms in critical areas [8].

In addition, the use of biological methods can reduce pollution, protect the ecological balance, and bring economic benefits. However, the effects of biological method may be very slow. Thus, we need to compare the suitability of using biological methods and artificial methods at the same time. The use of chemical reagents may be reduced with biological method, thereby reducing the negative effects of chemical methods. Based on previous research, we used the theory of impulsive differential equations [12-14] to develop a phytoplankton-fish model to investigate the feasibility of biological and artificial methods. Many researchers have studied some ecological systems with impulsive differential equations [15-22], including the use of biological and chemical controls.

In our model, a logistic growth function represents the gross rate of phytoplankton production. Based on previous studies [2, 23, 24], a Holling type-III function represents the interaction between the phytoplankton and the fish, that is, predation of the phytoplankton. Furthermore, based on the work of Wyatt and Horwood [25], Uye [26], and Levin and Segel [27], Truscott and Brindley [2] discussed the rationality of using a Holling type-III function to investigate the interaction between phytoplankton and zooplankton. We consider that the model is reasonable, although zooplankton is replaced by fish in our model. It is known that some filterfeeding fish feed on phytoplankton, so the population density of phytoplankton can control the rate of fish production. In this system, the loss of fish occurs via death and natural predation by higher trophic levels in the food web. Thus, we assume a linear loss of fish.

According to other studies [28, 29], some phytoplankton blooms may be controlled within a short period of time $(<24 \mathrm{~h})$ using artificial methods. In our model, we assume that the time unit is a day and the artificial and biological methods are modeled using impulsive differential equations. The model is described as follows:

$$
\begin{gathered}
\frac{d x}{d t}=r x\left(1-\frac{x}{K}\right)-\frac{a x^{2} y}{b+x^{2}}, \\
\frac{d y}{d t}=\frac{\varepsilon a x^{2} y}{b+x^{2}}-m y,
\end{gathered}
$$

$$
\begin{gathered}
\Delta x=-p x, \\
\Delta y=q y+\tau, \quad x=h,
\end{gathered}
$$

where $x$ denotes the phytoplankton population density, unit $\mu \mathrm{g} / \mathrm{L} ; y$ denotes the fish population density, unit $\mu \mathrm{g} / \mathrm{L} ; r$ is the intrinsic growth rate of phytoplankton population, $a$ is the maximum predation rate of the fish, $\varepsilon$ is the conversion efficiency, $K$ is the carrying capacity, $b$ is a half-saturation constant, and $m$ is the mortality and respiration rate of fish, while the term $\Delta x=-p x$, where $\Delta x=x\left(t^{+}\right)-x(t)$, represents artificial control and the term $\Delta y=(1+q) y+\tau$, where $\Delta y=$ $y\left(t^{+}\right)-y(t)$, represents biological control. The parameters $p \in(0,1)$ represent the control levels of artificial methods, $h>0$ denotes the critical value of a phytoplankton bloom, and $\tau \geq 0, q>0$ represents the release level of fish required to control phytoplankton, and we set $\Delta x=\alpha(x, y)=-p x$, $\Delta y=\beta(x, y)=q y+\tau$.

The rest of this paper is organized as follows. In Section 2, we provide some preliminary details, which are the theoretical basis of the following investigation. Next, we discuss the existence of an order-1 periodic solution, attractor, and bifurcation in Section 3, which provides a theoretical basis for the study of the biological method. In addition, some numerical results are presented in Section 4, which illustrate the validity of the theory. In the final section, we discuss the feasibility of the artificial and biological methods.

\section{Preliminaries}

Given the following autonomous system with impulsive control:

$$
\begin{array}{lll}
\frac{d x}{d t}=P(x, y), & \frac{d y}{d t}=Q(x, y), & (x, y) \notin N, \\
\Delta x=f(x, y), & \Delta y=g(x, y), & (x, y) \in N,
\end{array}
$$

where $t \in R,(x, y) \in R^{2}$, and $P, Q, f, g: R^{2} \rightarrow R, N \subset R^{2}$ are the set of impulses, it is assumed that $P, Q, f, g$ are all continuous with respect to $x, y$ in $R^{2}$, so the points in $N \subset R^{2}$ lie on a line. For each point $S(x, y) \in N, I: R^{2} \rightarrow R^{2}$ is defined:

$$
\begin{array}{r}
I(S)=S^{+}=\left(x^{+}, y^{+}\right) \in R^{2}, \\
x^{+}=x+f(x, y), \quad y^{+}=y+g(x, y) .
\end{array}
$$

Let $M=I(N)$ be the phase set of $N$, where $N \cap M=\phi$. System (2) is generally known as a semicontinuous dynamic system.

Definition 1. Let $\Gamma$ be an order-1 periodic solution of system (2), where $\Gamma$ is

(1) orbitally stable if $\forall \varepsilon>0, \exists p \in N, p \in \Gamma$, and $\exists \delta>0$ such that $\forall p_{1} \in \cup(p, \delta), \rho\left(\pi\left(p_{1}, t\right), \Gamma\right)<\varepsilon$ when $t>t_{0} ;$

(2) orbitally attractive if $\forall \varepsilon>0$ and $\forall p_{2} \in N, \exists T>0$ such that $\rho\left(\pi\left(p_{2}, t\right), \Gamma\right)<\varepsilon$ when $t>T+t_{0}$; 
(3) orbitally asymptotically stable if it is orbitally stable and orbitally attractive.

In the present study, $\cup(p, \delta)$ denotes the $\delta$-neighborhood of the point $p \in N, \rho\left(\pi\left(p_{1}, t\right), \Gamma\right)$ is the distance from $\pi\left(p_{1}, t\right)$ to $\Gamma$, and $\pi\left(p_{1}, t\right)$ is the solution of system (2) that satisfies the initial condition $\pi\left(p_{1}, t_{0}\right)=p_{1}$.

Definition 2. The phase plane is divided into two parts based on the trajectory of the differential equations that constitute the order-1 cycle. The section that contains the impulse line and the trajectory is known as the inside of the order-1 cycle.

Definition 3. Assuming that $M$ and $N$ are both straight lines, we define a new number axis $l$ on $N$. It is assumed that $N$ intersects with the $x$-axis at point $Q$. The point $Q$ is the origin on the number axis $l$, and both the positive direction and unit length are consistent with the coordinate axis $y$. For any point $A \in l$, let $l(A)=a$ be a coordinate of point $A$. Furthermore, supposing that the trajectory through point $A$ via $k$ th impulses intersects $N$ at point $B_{k}$, then set $l\left(B_{k}\right)=b_{k}$. The point $B_{k}$ is called the order- $k$ successor point of point $A$, and $F_{k}(A)$ is known as the order- $k$ successor function of point $A$, where $F_{k}(A)=l\left(B_{k}\right)-l(A)=b_{k}-a, k=1,2, \ldots$.

Lemma 4 (see [16]). The order-1 successor function $F_{1}(A)$ is continuous.

Lemma 5 (see [30]). The T-periodic solution $(x, y)=$ $(\xi(t), \eta(t))$ of the system

$$
\begin{aligned}
& \frac{d x}{d t}=P(x, y), \quad \frac{d y}{d t}=Q(x, y), \quad \phi(x, y) \neq 0, \\
& \Delta x=\xi(x, y), \quad \Delta y=\eta(x, y), \quad \phi(x, y)=0,
\end{aligned}
$$

is orbitally asymptotically stable if the Floquet multiplier $\mu$ satisfies the condition $|\mu|<1$, where

$$
\begin{aligned}
\mu= & \prod_{k=1}^{n} \Delta_{k} \\
& \times \exp \left[\int_{0}^{T}\left(\frac{\partial P}{\partial x}(\xi(t), \eta(t))+\frac{\partial Q}{\partial y}(\xi(t), \eta(t))\right) d t\right]
\end{aligned}
$$

with

$$
\begin{aligned}
\Delta_{k}= & \left(P_{+}\left(\frac{\partial \beta}{\partial y} \frac{\partial \phi}{\partial x}-\frac{\partial \beta}{\partial x} \frac{\partial \phi}{\partial y}+\frac{\partial \phi}{\partial x}\right)\right. \\
& \left.+Q_{+}\left(\frac{\partial \alpha}{\partial x} \frac{\partial \phi}{\partial y}-\frac{\partial \alpha}{\partial y} \frac{\partial \phi}{\partial x}+\frac{\partial \phi}{\partial y}\right)\right) \\
& \times\left(P \frac{\partial \phi}{\partial x}+Q \frac{\partial \phi}{\partial y}\right)^{-1},
\end{aligned}
$$

and $P, Q, \partial \alpha / \partial x, \partial \alpha / \partial y, \partial \beta / \partial x, \partial \beta / \partial y, \partial \phi / \partial x, \partial \phi / \partial y$, which are calculated for the points $\left(\xi\left(t_{k}\right), \eta\left(t_{k}\right)\right)$, $P_{+}=P\left(\xi\left(t_{k}^{+}\right), \eta\left(t_{k}^{+}\right)\right)$, and $Q_{+}=Q\left(\xi\left(t_{k}^{+}\right), \eta\left(t_{k}^{+}\right)\right)$, where $\phi(x, y)$ is a sufficiently smooth function so $\operatorname{grad} \phi(x, y) \neq 0$, and $t_{k}(k \in N)$ is the time of the kth jump.
Lemma 6 (see [31]). Let $F: R \times R \rightarrow R$ be a one-parameter family of the $C^{2}$ map that satisfies

(i) $F(0, \mu)=0$, (ii) $(\partial F / \partial x)(0,0)=1$, (iii) $\left(\partial^{2} F / \partial x \partial \mu\right)$ $(0,0)>0$, (iv) $\left(\partial^{2} F / \partial x^{2}\right)(0,0)<0$.

$F$ has two branches of fixed points for $\mu$ near zero. The first branch is $x_{1}(\mu)=0$ for all $\mu$. The second bifurcating branch $x_{2}(\mu)$ changes its value from negative to positive as $\mu$ increases through $\mu=0$ with $x_{2}(0)=0$. The fixed points of the first branch are stable if $\mu<0$ and unstable if $\mu>0$, whereas those of the bifurcating branch have the opposite stability.

\section{Main Results}

First, we consider the case of system (1) without an impulsive effect. We set $P(x, y)=r x(1-x / K)-a x^{2} y /\left(b+x^{2}\right), Q(x, y)=$ $\varepsilon a x^{2} y /\left(b+x^{2}\right)-m y$, and the equilibrium of system (1) without the impulsive effect implies $P(x, y)=0, Q(x, y)=0$, so we can obtain the following equilibrium under the conditions $\varepsilon a>m$ and $K>x^{*}$ :

(i) $E_{0}=(0,0)$ (total extinction);

(ii) $E_{1}=(K, 0)$ (extinction of the fish);

(iii) $E_{2}=\left(x^{*}, y^{*}\right)=\left(\sqrt{m b /(\varepsilon a-m)}, \operatorname{cbr}\left(K-x^{*}\right) /(\varepsilon a-\right.$ $\left.m) x^{*} K\right)$ (coexistence of phytoplankton and fish).

Both $x=0$ and $y=0$ are the trajectory of system (1) without an impulsive effect. Thus, the first quadrant is the invariant set.

Indeed, according to biological studies, the phytoplankton stable state $x^{*}$ should be smaller than the parameter $K$ because parameter $K$ is the maximum carrying capacity. Thus, we only need to assume the condition $\varepsilon a>m$. In system (1) without an impulsive effect, $a$ is the maximum predation rate of the fish, $\varepsilon$ is the maximum conversion, and $\varepsilon a$ denotes the maximum growth rate. If condition $\varepsilon a \leq m$ holds, that is, the growth rate of the fishes is below the mortality rate of the fishes, then the fishes may become extinct. In system (1) without an impulsive effect, based on a direct calculation, the equilibrium $E_{0}$ is always saddle. Thus, the equilibrium $E_{1}$ is locally asymptotical stable when the condition $\varepsilon a \leq m$ holds. Then, the solution of system (1) without an impulsive effect will converge toward the equilibrium $E_{1}$ in a certain field; that is, the fishes will become extinct and the phytoplankton will bloom. Phytoplankton blooms are observed frequently, but the growth rate of fishes being less than the mortality rate of fishes is usually rare in real life. Therefore, we assume that condition $\varepsilon a>m$ holds in full. Obviously, $y=f(x)=$ $r(1-x / K)\left(b+x^{2}\right) / a x^{2}$ is a vertical line and $x=\sqrt{m b /(\varepsilon a-m)}$ is a horizontal isocline. By direct calculation, the equilibrium $E^{*}$ is locally asymptotically stable under the condition ( $\varepsilon a-$ $2 m) K>-2 m x^{*}$ and the index of the equilibrium $E^{*}$ is +1 . Throughout this paper, we assume that the condition $(a \varepsilon-$ $2 m) K>-2 m x^{*}$ always holds based on ecological studies, and we let $N(x=h)$ and $M(x=(1-p) h)$ be the impulsive set and phase set, respectively, with the point $O=N \cap\{y=f(x)\}$, the point $H=M \cap\{y=f(x)\}$, and $h<x_{*}$. For the case where $x_{*}<h<K$, similar results can be obtained using the same method. 


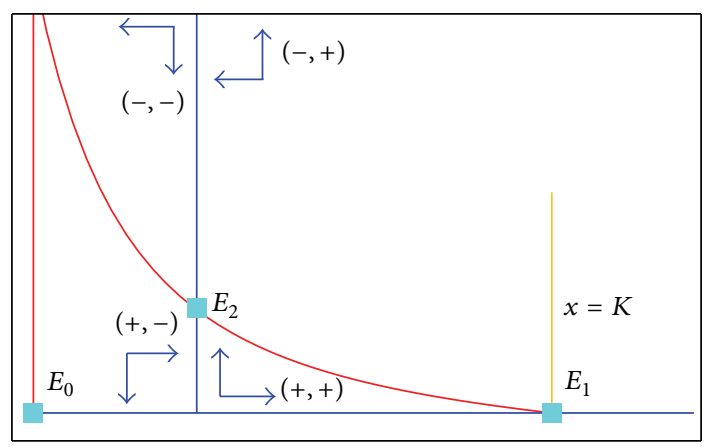

$-(d x / d t)=0$

$-(d y / d t)=0$

FIgURE 1: Direction of the trajectory in system (1) without an impulsive effect in the first quadrant.

From $f(x)$, we know that the line $x=0$ is the asymptote of the curve $y=f(x)$, and $y>0$ when $x<K, y=0$ when $x=K, y<0$ when $x>K$. In addition, when $x<K$, the function $f(x)$ is a monotonically decreasing function. The first quadrant is split into four parts by the isocline (see Figure 1).

\subsection{Existence of an Order-1 Periodic Solution for System (1)}

3.1.1. The Case Where $\tau=0$. In this subsection, we derive some basic properties of the following subsystem of system (1), where fish $y(t)$ are absent:

$$
\begin{gathered}
\frac{d x}{d t}=r x\left(\frac{K-x}{K}\right) \quad x<h \\
\Delta x=-p x \quad x=h .
\end{gathered}
$$

Setting $x_{0}=x(0)=(1-p) h$ produces the following solution of system (7): $x(t)=K(1-p) h \exp (r(t-n T)) /(K-(1-$ $p) h+(1-p) h \exp (r(t-n T)))$. If we let $T=(1 / r) \ln ((K-(1-$ $p) h) /(K-h)(1-p)), x(T)=h$, and $x\left(T^{+}\right)=(1-p) h$, this means that system (1) has the following semitrivial periodic solution:

$$
\begin{gathered}
x(t)=\frac{K(1-p) h \exp (r(t-n T))}{K-(1-p) h+(1-p) h \exp (r(t-n T))} \\
y(t)=0,
\end{gathered}
$$

where $t \in(n T,(n+1) T], n \in N$, which is implied by $(\xi(t), 0)$.

Thus, the following theorem is obtained.

Theorem 7. There exists a semitrivial periodic solution (8) to system (1), which is orbitally asymptotically stable if

$$
0<q<\Psi^{-1}-1
$$

where $\Psi=((K-(1-p) h) /(K-h)(1-p))^{-m / r} \eta_{1} \eta_{2}$

$$
\begin{aligned}
& \eta_{1}=\left(\frac{(K-(1-p) h)^{2}\left(b+h^{2}\right)}{\left(b+((1-p) h)^{2}\right)(K-h)^{2}}\right)^{\varepsilon a K^{2} / 2 r\left(b+K^{2}\right)}, \\
& \eta_{2}=\exp \left(-\frac{\varepsilon a K \sqrt{b}}{r\left(b+K^{2}\right)}\right. \\
& \quad\left(\arctan \left(\frac{(h K+b)}{\sqrt{b}(K-h)}\right)\right. \\
&\left.\left.\quad-\arctan \left(\frac{K(1-p) h+b}{\sqrt{b}(K-(1-p) h)}\right)\right)\right) .
\end{aligned}
$$

Proof. It is known that $P(x, y)=r x(1-x / K)-a x^{2} y /(b+$ $\left.x^{2}\right), Q(x, y)=\varepsilon a x^{2} y /\left(b+x^{2}\right)-m y, \alpha(x, y)=-p x$, $\beta(x, y)=q y, \phi(x, y)=x-h,(\xi(T), \eta(T))=(h, 0)$, and $\left(\xi\left(T^{+}\right), \eta\left(T^{+}\right)\right)=((1-p) h, 0)$. Using Lemma 5 and a straightforward calculation, it is possible to obtain

$$
\begin{gathered}
\frac{\partial P}{\partial x}=r\left(1-\frac{2}{K} x\right)-\frac{2 a b x y}{\left(b+x^{2}\right)^{2}}, \quad \frac{\partial Q}{\partial y}=\frac{\varepsilon a x^{2}}{b+x^{2}} \\
\frac{\partial \alpha}{\partial x}=-p, \quad \frac{\partial \alpha}{\partial y}=0 ; \quad \frac{\partial \beta}{\partial x}=0, \\
\frac{\partial \beta}{\partial y}=q ; \quad \frac{\partial \phi}{\partial x}=1, \quad \frac{\partial \phi}{\partial y}=0, \\
\Delta_{1}=\left(P_{+}\left(\frac{\partial \beta}{\partial y} \frac{\partial \phi}{\partial x}-\frac{\partial \beta}{\partial x} \frac{\partial \phi}{\partial y}+\frac{\partial \phi}{\partial x}\right)\right. \\
\left.+Q+\left(\frac{\partial \alpha}{\partial x} \frac{\partial \phi}{\partial y}-\frac{\partial \alpha}{\partial y} \frac{\partial \phi}{\partial x}+\frac{\partial \phi}{\partial y}\right)\right) \\
\times\left(P \frac{\partial \phi}{\partial x}+Q \frac{\partial \phi}{\partial y}\right)^{-1}, \\
=\frac{P^{+}\left(\xi\left(T^{+}\right), \eta\left(T^{+}\right)\right)(1+q)}{P(\xi(T), \eta(T))} \\
=(1-p)(1+q) \frac{K-(1-p) h}{K-h} .
\end{gathered}
$$

Furthermore,

$$
\begin{aligned}
& \exp \left[\int_{0}^{T}\left(\frac{\partial P}{\partial x}(\xi(t), \eta(t))+\frac{\partial Q}{\partial y}(\xi(t), \eta(t))\right) d t\right] \\
& =\exp \left[\int_{0}^{T}\left(r\left(1-\frac{2}{K} \xi(t)\right)+\frac{\varepsilon a(\xi(t))^{2}}{b+(\xi(t))^{2}}-m\right) d t\right]
\end{aligned}
$$




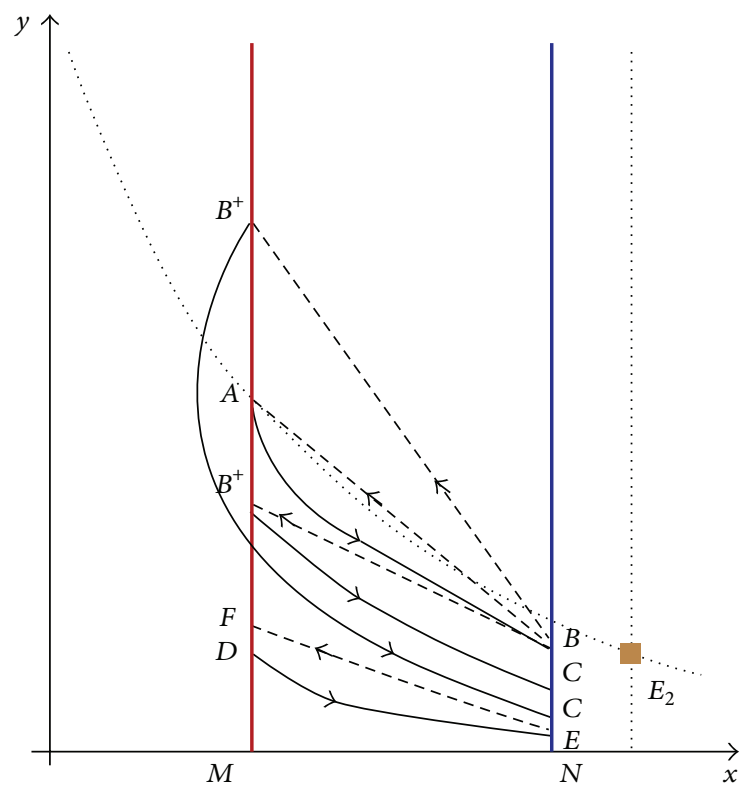

(a)

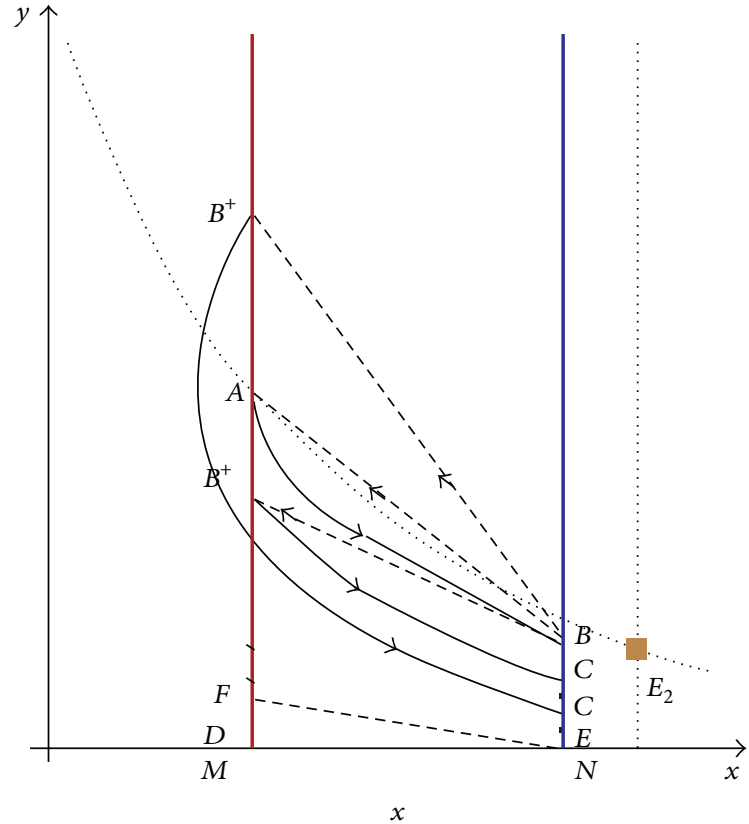

(b)

FIgURE 2: The proof of Theorems 9 and 10 .

$$
\begin{array}{r}
=\left(\frac{K-(1-p) h}{(K-h)(1-p)}\right)^{r-m / r}\left(\frac{K-h}{K-(1-p) h}\right)^{2} \eta_{1} \eta_{2}, \\
\eta_{1}=\left(\frac{(K-(1-p) h)^{2}\left(b+h^{2}\right)}{\left(b+((1-p) h)^{2}\right)(K-h)^{2}}\right)^{\varepsilon a K^{2} / 2 r\left(b+K^{2}\right)} \\
\eta_{2}=\exp \left(-\frac{\varepsilon a K \sqrt{b}}{r\left(b+K^{2}\right)},\right. \\
\quad \times\left(\arctan \left(\frac{(h K+b)}{\sqrt{b}(K-h)}\right)\right. \\
\left.\quad-\arctan \left(\frac{K(1-p) h+b}{\sqrt{b}(K-(1-p) h)}\right)\right) .
\end{array}
$$

Therefore, it is possible to obtain the Floquet multiplier $\mu$ by direct calculation as follows:

$$
\begin{aligned}
\mu= & \prod_{k=1}^{n} \Delta_{k} \\
& \times \exp \left[\int_{0}^{T}\left(\frac{\partial P}{\partial x}(\xi(t), \eta(t))+\frac{\partial Q}{\partial y}(\xi(t), \eta(t))\right) d t\right] \\
= & (1+q) \Psi .
\end{aligned}
$$

Thus, $|\mu|<1$, if (9) holds. This completes the proof.
Remark 8. If $q^{*}=\Psi^{-1}-1$, a bifurcation may occur when $q=q^{*}$ for $|\mu|=1$, whereas a positive periodic solution may emerge if $q>q^{*}$.

Theorem 9. There exists a positive order-1 periodic solution to system (1) if $q>q^{*}$, where the semitrivial periodic solution is orbitally unstable.

Proof. Because $h<x^{*}$, both $M$ and $N$ are in the left $E_{2}$. The trajectory that passes through point $A$, where $A=M \cap\{y=$ $f(x)\}$, tangents to $M$ at point $A$ and intersects $N$ at point $B$. Thus, there may be three possible cases for the phase point $\left(B^{+}\right)$for point $B$, as follows (see Figure 2(a)).

Case $1\left(y_{A}=y_{B^{+}}\right)$. In this case, it is obvious that $A B A$ is an order-1 periodic solution.

Case $2\left(y_{A}<y_{B^{+}}\right)$. The point $B^{+}$is the order- 1 successor point of point $A$, so the order-1 successor function of point $A$ is greater than zero; that is, $F_{1}(A)=y_{B^{+}}-y_{A}>0$. In addition, the trajectory with the initial point $B^{+}$intersects impulsive set $N$ at point $C$ and reaches $C^{+}$via the impulsive effect. Due to the disjointedness of the trajectory and impulsive line in system (1), it is easy to see that point $C^{+}$is located below point $B^{+}$. Therefore, the successor function $F_{1}\left(B^{+}\right)<0$. According to Lemma 4 , there exists a point $G \in M$, such that $F_{1}(G)=0$; hence, there exists an order-1 periodic solution.

Case $3\left(y_{A}>y_{B^{+}}\right)$. According to $y_{A}>y_{B^{+}}$, the order1 successor point of point $A$ is located below point $A$, so $F_{1}(A)<0$. If we suppose that $p_{0}$ is a crossover point of the semitrivial periodic solution and impulsive set, because the semitrivial periodic solution is orbitally unstable, then 
there exists a point $H_{0} \in \mathrm{U}\left(p_{0}, \delta\right)$ such that $F_{1}\left(H_{0}\right)>0$. According to Lemma 4 , there exists a point $K_{0} \in M$, such that $F_{1}\left(K_{0}\right)=0$. Therefore, there exists an order-1 periodic solution in system (1).

This completes the proof.

3.1.2. The Case $\tau>0$. In this case, we suppose that $h<x^{*}$ and the following theorem is described.

Theorem 10. There exists a positive order-1 periodic solution for system (1) if $\tau>0$ and $h<x^{*}$.

Proof. The method for this proof is similar to the method for Theorem 9 (see Figure 2(b)). The main difference is the proof for the case $y_{A}>y_{B^{+}}$. Suppose that point $D$ is a crossover point for a semitrivial periodic solution and a phase set. The trajectory with initial point $D$ intersects the impulsive set at point $E$. Obviously, $y_{D}=y_{E}=0$. Because $\tau>0$, $y_{F}=(1+q) y_{E}+\tau>0=y_{D}$. Thus, there exists a positive order-1 periodic solution for system (1), which completes the proof.

Remark 11. If there exists a positive order-1 periodic solution $\Gamma$ for system (1) when $h<x^{*}$, set point $S=\Gamma \cap N$, then $y_{S}<y_{O}$.

In summary, system (1) has a stable semitrivial periodic solution or a positive order-1 periodic solution when $\tau \geq 0$. Furthermore, using the analogue of the Poincaré criterion, the stability of the positive order-1 periodic solution is obtained.

Theorem 12. For any $\tau>0, q>0$ or $\tau=0, q \geq q^{*}$, the order -1 periodic solution of system (1) is orbitally asymptotically stable if the following condition holds:

$$
\left|\frac{P\left((1-p) h,(1+q) \eta_{0}+\tau\right)}{P\left(h, \eta_{0}\right)}(1+q) \exp \left(\int_{0}^{T} G(t) d t\right)\right|
$$

$$
<1
$$

where $G(t)=(\partial P / \partial A)(\xi(t), \eta(t))+(\partial Q / \partial F)(\xi(t), \eta(t))$.

Proof. We suppose that the period of the order-1 periodic solution is $T$, so the order-1 periodic solution intersects with the impulsive set at $P\left(h, \eta_{0}\right)$ and the phase set at $P^{+}((1-$ $\left.p) h,(1+q) \eta_{0}+\tau\right)$. Let $(\xi(t), \eta(t))$ be the expression of the order-1 periodic solution. The difference between this case and the case in Theorem 7 is that $(\xi(T), \eta(T))=$ $\left(h, \eta_{0}\right),\left(\xi\left(T^{+}\right), \eta\left(T^{+}\right)\right)=\left((1-p) h,(1+q) \eta_{0}+\tau\right)$, while the others are the same. Thus, we have

$$
\begin{gathered}
\Delta_{1}=\frac{P\left((1-p) h,(1+q) \eta_{0}+\tau\right)}{P\left(h, \eta_{0}\right)}(1+q) \\
\mu_{2}=\Delta_{1} \exp \left(\int_{0}^{T} G(t) d t\right) .
\end{gathered}
$$

According to condition (14), $\left|\mu_{2}\right|<1$, so the order-1 periodic solution is orbitally stable according to the analogue of the Poincaré criterion. This completes the proof.
Theorem 13. In system (1), if the conditions $(1+q) y_{O}+\tau<y_{H}$ and $h<x^{*}$ hold, then the set $\Omega$, where $\Omega=[(1-p) h, h] \times$ $[0, f(x)](x \in[(1-p) h, h])$, is an attractor. There is also no order- $k(k \geq 2)$ periodic solution.

Proof. In the region $\Phi=(0, h] \times(0,+\infty), \forall\left(x_{0}, y_{0}\right) \in \Phi$, there exists a time $t_{1}$ such that the trajectory $\varphi\left(t ; x_{0}, y_{0}\right)$ of system (1) reaches the impulsive set $N$ at point $L$ when $t=t_{1}$ and $y_{L}<y_{O}$. Via the impulsive effect, the trajectory reaches the phase set $M$, and then $\lim _{t \rightarrow+\infty}$ inf $x(t) \geq(1-p) h$; in addition, $\lim _{t \rightarrow+\infty}$ sup $x(t) \leq h$. Because $(1+q) y_{O}+\tau<$ $y_{H},(1+q) y_{L}+\tau<y_{H}$. Furthermore, the trajectory of the differential equation is not intersectant. From system (1), we know that the impulsive line is also not intersectant. Thus, $\lim _{t \rightarrow+\infty} \sup y(t) \leq f(x),(x \in[(1-p) h, h])$. Therefore, the set $\Omega$ is a global attractor in the region $\Phi$.

Supposing that there exists an order- $k(k \geq 2)$ periodic solution to system (1), the initial point of the order- $k$ periodic solution must be above the point $H$. We set the initial point as $J$ andthen $y_{J}>y_{H}$, and we suppose that the $k$ th point of the order- $k$ periodic solution that reaches the impulsive set is point $Z$. Then, $(1+q) y_{Z}+\tau=y_{J}$ and $y_{Z}<y_{O}$. However, $(1+q) y_{O}+\tau<y_{H}$. Therefore, this is a contradiction. Hence, there is no order- $k(k \geq 2)$ periodic solution.

This completes the proof.

\subsection{The Bifurcation}

3.2.1. Transcritical Bifurcation. In this subsection, we discuss the bifurcation near the semitrivial periodic solution. The following Poincaré map $P$ is used:

$$
y_{k}^{+}=(1+q) \sigma\left(y_{k-1}^{+}\right)
$$

where we choose section $S_{0}=(1-p) h$ as a Poincaré section. If we set $0 \leq u=y_{k}^{+}$at a sufficiently small value, the map can be written as follows:

$$
u \longmapsto(1+q) \sigma(u) \equiv G(u, q) .
$$

Using Lemma 6, the following theorem can be obtained.

Theorem 14. A transcritical bifurcation occurs when $q=q^{*}$, $\tau=0$. Correspondingly, system (1) has a stable positive periodic solution when $q \in\left(q^{*}, q^{*}+\delta\right)$ with $\delta>0$.

Proof. The values of $\sigma^{\prime}(u)$ and $\sigma^{\prime \prime}(u)$ must be calculated at $u=$ 0 where $0 \leq u \leq u_{0}$. In this case, $u_{0}=r(1-h / K)\left(b+h^{2}\right) / a h$. Thus, system (1) can be transformed as follows:

$$
\frac{d y}{d x}=\frac{Q(x, y)}{P(x, y)}
$$

where $P(x, y)=r x(1-x / K)-a x^{2} y /\left(b+x^{2}\right), Q(x, y)=$ $\varepsilon a x^{2} y /\left(b+x^{2}\right)-m y$.

Let $\left(x, y\left(x ; x_{0}, y_{0}\right)\right)$ be an orbit of system (18) and $x_{0}=$ $(1-p) h, y_{0}=u, 0 \leq u \leq u_{0}$. Then,

$$
\begin{array}{r}
y(x ;(1-p) h, u) \equiv y(x, u), \\
(1-p) h \leq x \leq h, \quad 0 \leq u \leq u_{0} .
\end{array}
$$


Using (19),

$$
\begin{aligned}
& \frac{\partial y(x, u)}{\partial u}=\exp \left[\int_{(1-p) h}^{x} \frac{\partial}{\partial y}\left(\frac{Q(s, y(s, u))}{P(s, y(s, u))}\right) d s\right], \\
& \frac{\partial^{2} y(x, u)}{\partial u^{2}} \\
& =\frac{\partial y(x, u)}{\partial u} \int_{(1-p) h}^{x} \frac{\partial^{2}}{\partial y^{2}}\left(\frac{Q(s, y(s, u))}{P(s, y(s, u))}\right) \frac{\partial y(s, u)}{\partial u} d s,
\end{aligned}
$$

it can be deduced simply that $\partial y(x, u) / \partial u>0$ and

$$
\begin{aligned}
\sigma^{\prime}(0) & \frac{\partial y(h, 0)}{\partial u} \\
= & \exp \left(\int_{(1-p) h}^{h} \frac{\partial}{\partial y}\left(\frac{Q(s, y(s, 0))}{P(s, y(s, 0))}\right) d s\right) \\
= & \exp \left(\int_{(1-p) h}^{h} \frac{\left(\varepsilon a s^{2} /\left(b+s^{2}\right)\right)-m}{r s(1-(s / K))} d s\right) \\
= & \left(\frac{K-(1-p) h}{(K-h)(1-p)}\right)^{-m / r} \\
& \times\left(\frac{(K-(1-p) h)^{2}\left(b+h^{2}\right)}{\left(b+((1-p) h)^{2}\right)(K-h)^{2}}\right) \\
& \times \exp \left(\frac{-\varepsilon a K \sqrt{b} / 2 r\left(b+K^{2}\right)}{r\left(K^{2}+b\right)}\right. \\
& \times\left(\arctan \left(\frac{(h K+b)}{\sqrt{b}(K-h)}\right)\right. \\
& \left.-\arctan \left(\frac{K(1-p) h+b}{\sqrt{b}(K-(1-p) h)}\right)\right) .
\end{aligned}
$$

Thus, $\sigma^{\prime}(0)=\Psi$.

Furthermore,

$$
\sigma^{\prime \prime}(0)=\sigma^{\prime}(0) \int_{(1-p) h}^{h} m(s) \frac{\partial y(s, 0)}{\partial u} d s,
$$

where $m(s)=\left(\partial^{2} / \partial y^{2}\right)(Q(s, y(s, 0)) / P(s, y(s, 0)))=$ $\left(\varepsilon a s^{2} /\left(b+s^{2}\right)\right)-m /(r s(1-(s / K)))^{3}, s \in[(1-p) h, h]$. Because $s \leq h<x^{*}$, it can be determined that $m(s)<0, s \in$ $[(1-p) h, h]$. Therefore,

$$
\sigma^{\prime \prime}(0)<0 .
$$

The next step is to check whether the following conditions are satisfied.

(a) It is easy to see that $G(0, q)=0, q \in(0, \infty)$. (b) Using (21), $\partial G(0, q) / \partial u=(1+q) \sigma^{\prime}(0)=(1+q) \Psi$, which yields $\partial G\left(0, q^{*}\right) / \partial u=1$. This means that $\left(0, q^{*}\right)$ is a fixed point with an eigenvalue of 1 in map (16).

(c) Because (21) holds, $\partial^{2} G\left(0, q^{*}\right) / \partial u \partial q=\sigma^{\prime}(0)>0$.

(d) Finally, inequality (23) implies that $\partial^{2} G\left(0, q^{*}\right) / \partial u^{2}=$ $\left(1+q^{*}\right) \sigma^{\prime \prime}(0)<0$.

These conditions satisfy the conditions of Lemma 6 . This completes the proof.

In the region $\Phi=(0, h] \times(0,+\infty), d y / d t<0$ always holds. Thus, $\forall\left(x_{0}, y_{0}\right) \in \Phi$, the trajectory $\varphi\left(x ; x_{0}, y_{0}\right)$ first intersects the phase set $M$ at point $\rho$ and the impulsive set $N$ at point $\pi$, respectively; then, $y_{\pi}<y_{\rho}$. Therefore, there is always a certain pair of $(q, \tau)$, such that $y_{\rho}=(1+q) y_{\pi}+\tau$ for $q>0, \tau \geq 0$. Thus, in the region $\Phi$, there exists an order-1 periodic solution in system (1). In addition, the order1 periodic solution is unique when the initial point of the order-1 periodic solution in the phase set is above point $H$. This is obvious because of the disjointedness of the trajectory of the differential equation.

3.2.2. The Bifurcation of the Order-1 Periodic Solution. In this subsection, we discuss the bifurcation of an order-1 periodic solution with variable parameters. The following theorem is required.

Theorem 15. The rotation direction of the pulse line is clockwise when $q$ changes from $q=0$ to $q>0$.

Proof. Let $\theta$ be the angle of the pulse line and the $x$ axis. Then, $\tan \theta=\Delta y / \Delta x=\beta(x, y) / \alpha(x, y)$, so $\theta=$ $\tan ^{-1}(\alpha(x, y) / \beta(x, y))$. Furthermore, $\partial \theta / \partial q=\left(1 /\left(\alpha^{2}+\right.\right.$ $\left.\left.\beta^{2}\right)\right)\left|\begin{array}{cc}\alpha & \beta \\ \partial \alpha / \partial q \partial \beta / \partial q\end{array}\right|=1 /\left(\alpha^{2}+\beta^{2}\right)(-p x y)<0$. Therefore, $\theta$ is a monotonically decreasing function of $q$. This completes the proof.

The existence of an order-1 periodic solution was proved in the previous analysis, so we assume that there exists an order-1 periodic solution $\Gamma_{*}$ when $q=q_{1}$ and $\tau \geq 0$, where the crossover points of $\Gamma_{*}$ for $M$ and $N$ are points $R$ and $Z$, respectively. Thus, the following theorem can be stated.

Theorem 16. In system (1), supposing that there exists an order-1 periodic solution when $q=q_{1}, \tau>0$ and $y_{R}>y_{H}$, then there exists a unique order-1 periodic solution $\Gamma_{* *}$ inside the order-1 periodic solution $\Gamma_{*}$ when $q=q_{1}-\vartheta$, if $\vartheta>0$ is sufficiently small. In addition, if $\Gamma_{*}$ is orbitally asymptotically stable, then $\Gamma_{* *}$ is orbitally asymptotically stable.

Proof. The order-1 periodic solution breaks when $q$ changes (see Figure 3(a)). According to Theorem 15, point $B_{1}$, which is the phase point of point $Z$, is located below point $R$ when $q=q_{1}-\vartheta$ and $\vartheta>0$ is sufficiently small. Because $y^{+}=$ $y+q y+\tau$ (in this case, $y>0$ ) is a monotonically increasing and continuing function of $q$, there exists $\vartheta_{1}>0$ such that $y_{C_{1}}<y_{H}<y_{B_{1}}<y_{R}$ when $q=q_{1}-\vartheta_{1}$. Figure $3($ a) shows that point $B_{1}$ is the order-1 successor point of point $R$, while point $A_{1}$ is the order- 1 successor point of point $B_{1}$, so $F_{1}(R)<0$, 


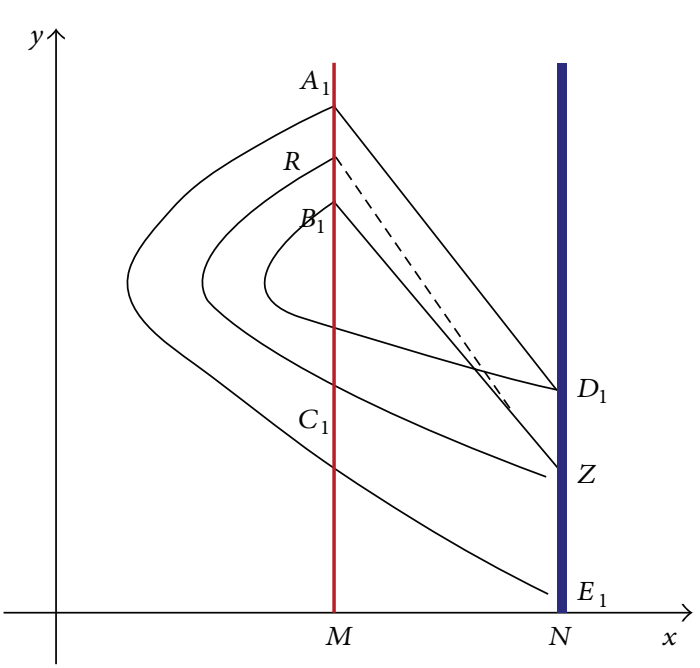

(a)

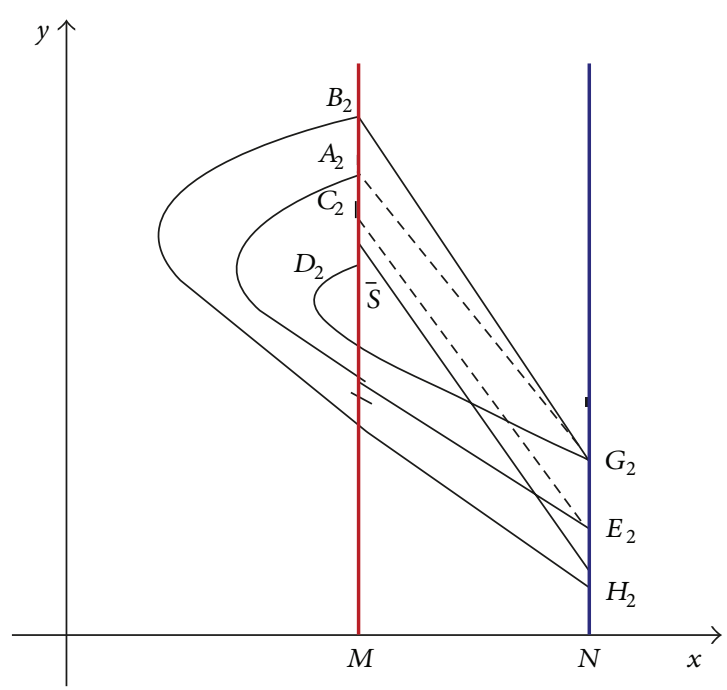

(b)

FIgURE 3: The proof of Theorem 16.

$F_{1}\left(B_{1}\right)>0$. Therefore, there exists a point $K_{1}$ between point $R$ and $B_{1}$ such that $F_{1}\left(K_{1}\right)=0$. According to the disjointedness of the differential trajectories, the order-1 periodic solution $\Gamma_{* *}$ is inside the order-1 periodic solution $R C_{1} Z R$.

Next, the orbital stability can be established based on the following proof (see Figure 3(b)).

The order-1 periodic solution $R C_{1} Z R$ is orbitally asymptotically stable, so, according to, the disjointedness of the pulse line, there exists a point $S$ between point $R$ and $H$ such that $F_{1}(S)>0, F_{2}(S)>0$. We suppose that the reduction in $q_{1}$ is $\vartheta>0$.

If $\vartheta=0$, point $B_{2}$ is the order-1 successor point of point $S$ and point $D_{2}$ is the order- 2 successor point of point $S$. Because of $y_{B_{2}}=\left(1+q_{1}\right) y_{G_{2}}+\tau, y_{D_{2}}=\left(1+q_{1}\right) y_{H_{2}}+\tau, F_{1}(S)=$ $y_{B_{2}}-y_{S}=\left(1+q_{1}\right) y_{G_{2}}+\tau-y_{S}>0, F_{2}(S)=y_{D_{2}}-y_{S}=$ $\left(1+q_{1}\right) y_{H_{2}}+\tau-y_{S}>0$.

If $\vartheta>0$, the order- 1 and order- 2 successor points of point $S$ are points $A_{2}$ and $C_{2}$, respectively, where $y_{A_{2}}=\left(1+q_{1}-\right.$ 9) $y_{G_{2}}+\tau$, $y_{C_{2}}=\left(1+q_{1}-\vartheta\right) y_{E_{2}}+\tau$. Therefore, $F_{1}(S)=$ $y_{A_{2}}-y_{S}=\left(1+q_{1}-\vartheta\right) y_{G_{2}}+\tau-y_{S}, F_{2}(S)=y_{C_{2}}-y_{S}=\tau-$ $y_{S}+\left(1+q_{1}-\vartheta\right) y_{E_{2}}$, and in order to distinguish the successor functions between $\vartheta=0$ and $\vartheta>0$, we set $F_{1}(S)=F_{1}^{1}(S)$ and $F_{2}(S)=F_{2}^{2}(S)$.

Therefore, we have the following: $F_{1}^{1}(S)=\left(1+q_{1}-9\right) y_{G_{2}}+$ $\tau-y_{S}=\left(1+q_{1}\right) y_{G_{2}}+\tau-y_{S}-\vartheta y_{G_{2}}$, because $\left(1+q_{1}\right) y_{G_{2}}+\tau-y_{S}>$ 0 , so $F_{1}^{1}(S)>0$ when $0<\vartheta<\left(\left(1+q_{1}\right) y_{G_{2}}+\tau-y_{S}\right) / y_{G_{2}} \stackrel{\text { define }}{\longrightarrow}$ $\vartheta_{11}$.

In addition,

$$
\begin{aligned}
& F_{2}(S)-F_{2}^{2}(S) \\
& \quad=\left(1+q_{1}\right) y_{H_{2}}+\tau-y_{S}-\left(1+q_{1}-\vartheta\right) y_{E_{2}}-\tau+y_{S} .
\end{aligned}
$$

Obviously, $F_{2}(S)-F_{2}^{2}(S)<0$ when $0<9<(1+$ $\left.q_{*}\right)\left(y_{E_{2}}-y_{H_{2}}\right) / y_{H_{2}} \stackrel{\text { define }}{\longrightarrow} \vartheta_{22}$, where $y_{E_{2}}>y_{H_{2}}$ from
Figure 3(b). If we set $\vartheta^{*}=\min \left(\vartheta_{11}, \vartheta_{22}\right)$, then $F_{1}^{1}(S)>0$ and $F_{2}^{2}(S)>0$ when $\vartheta \in\left(0, \vartheta^{*}\right)$. Next, we will prove that the trajectory initialization point $S$ is attracted by $\Gamma_{* *}$. We set $d_{n}=F_{2 n-1}^{2 n-1}(S)-F_{2 n}^{2 n}(S)(n \in N)$, where $F_{2 n}^{2 n}(S)$ denotes the order-2n successor of point $S$. hold:

Because $F_{1}^{1}(S)>0$ and $F_{2}^{2}(S)>0$, the following results

$$
\begin{gathered}
F_{1}^{1}(S)>F_{3}^{3}(S)>\cdots>F_{2 n-1}^{2 n-1}(S), \\
F_{2}^{2}(S)<F_{4}^{4}(S)<\cdots<F_{2 n}^{2 n}(S), \\
F_{2 n-1}^{2 n-1}(S)>F_{2 n}^{2 n}(S), \\
0<d_{n}=\alpha_{n-1} d_{n-1}, \quad\left(0<\alpha_{n}<1\right) .
\end{gathered}
$$

When $n=1,2,3$, the expression (25a), (25b), (25c), (25d) obviously holds. Suppose that (25a), (25b), (25c), (25d) holds when $n=j$. Now, we set $n=j+1$. For the trajectory initialization point order- $2 j-1$ successor point of point $S$, its order-1 successor point is the order- $2 j$ successor point of point $S$, its order-2 successor point is the order- $2 j+1$ successor point of point $S$, and its order-3 successor point is the order- $2 j+2$ successor point of point $S$. Thus, it is obvious that $F_{2 j-1}^{2 j-1}>F_{2 j+1}^{2 j+1}, F_{2 j}^{2 j}<F_{2 j+2}^{2 j+2}, F_{2 j+1}^{2 j+1}(S)>F_{2 j+2}^{2 j+2}(S)$, and $d_{j+1}<d_{j}$. Therefore, (25a), (25b), (25c), (25d) holds. Moreover, based on $0<d_{n}=\alpha_{n-1} d_{n-1},\left(0<\alpha_{n}<1\right)$, it is known that $d_{n}=\alpha_{1}, \ldots, \alpha_{n-1} d_{1}$. Because $0<\alpha_{n}<1$, then $\lim _{n \rightarrow+\infty} d_{n}=0$. Thus, $\pi(t, S) \rightarrow \Gamma_{* *}, \quad t \rightarrow+\infty$, where $\pi(t, S)$ is the trajectory initialization point $S$ in system (1).

Similarly, if $y_{S}>y_{R}$, then there exist $\vartheta_{* *}$ such that $F_{1}^{1}(S)<$ 0 and $F_{2}^{2}(S)<0$ when $\vartheta \in\left(0, \vartheta^{* *}\right)$. The following results hold.

$$
F_{1}^{1}(S)<F_{3}^{3}(S)<\cdots<F_{2 n-1}^{2 n-1}(S),
$$




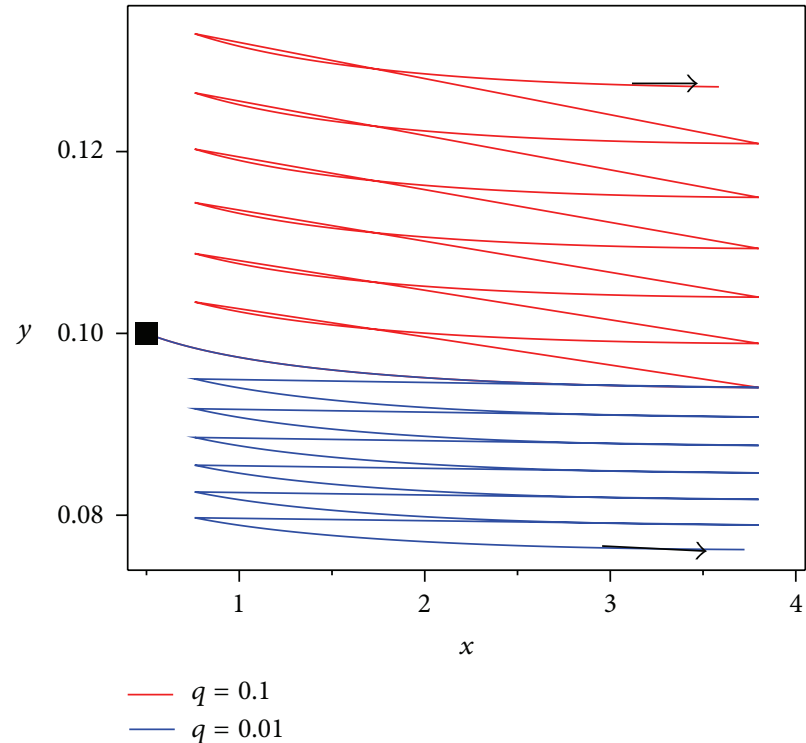

(a)

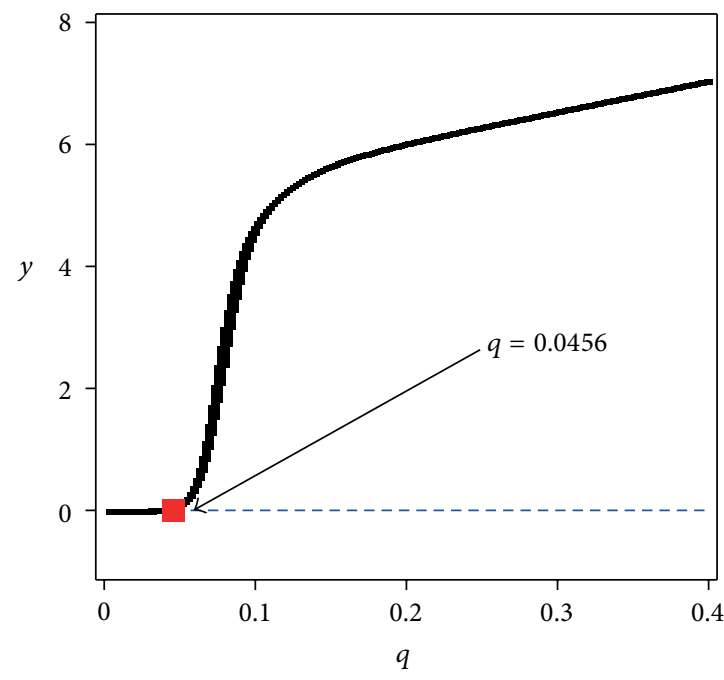

(b)

FIGURE 4: (a) Trajectories based on the initial point $(0.5,0.1)$ in system $(1)$, where the black symbol denotes the initial point $(0.5,0.1)$; the red curve denotes the trajectory when $q=0.1$; the blue curve denotes the trajectory when $q=0.01$; and the arrow denotes the direction of the trajectory. (b) Bifurcation diagram of system (1), where the red symbol denotes the bifurcation point; the dashed line implies the instability of the semitrivial solution; and the black solid line represents the maximum value of population $y$, which is stable, where $p=0.8, \tau=0$.

$$
\begin{gathered}
F_{2}^{2}(S)>F_{4}^{4}(S)>\cdots>F_{2 n}^{2 n}(S), \\
F_{2 n}^{2 n}(S)>F_{2 n-1}^{2 n-1}(S), \\
0>d_{n}=\alpha_{n-1} d_{n-1}, \quad\left(0<\alpha_{n}<1\right) .
\end{gathered}
$$

Then, $\pi(t, S) \rightarrow \Gamma_{* *}, \quad t \rightarrow+\infty$, where $\pi(t, S)$ is the trajectory initialization point $S$ in system (1).

Similarly, using the disjointedness of the trajectory of the differential equation and the impulsive line, we set $S_{*}$ as the arbitrary point between point $R$ and point $S$ in phase line, and we can prove $\pi(t, S) \rightarrow \Gamma_{* *}, t \rightarrow+\infty$. Thus, $\Gamma_{* *}$ is orbitally attractive.

Because $\Gamma_{* *}$ is unique and orbitally attractive, there exists a $T_{0}$ such that $\rho\left(\pi\left(t, p_{0}\right), \Gamma_{* *}\right)<\varepsilon_{0}$ for $\forall \varepsilon_{0}>0$ and $p_{0} \in$ $M\left(p_{0}>y_{H}\right)$, set $p_{* *}=\Gamma_{* *} \cap M$. In addition, there must be a $T_{1} \geq T_{0}$, such that $\pi\left(T_{1}, p_{0}\right)=\pi\left(T_{1}, p_{0}\right) \cap M$, if we set $\pi\left(T_{1}, p_{0}\right)=p_{1}$. We arbitrarily take the point $p_{2}$ between point $p_{* *}$ and point $p_{1}$, then $\rho\left(\pi\left(t, p_{2}\right), \Gamma_{* *}\right)<\varepsilon_{0}$ when $t \geq t_{0}$. If we set $\delta=\left|y_{p_{1}}-y_{p_{* *}}\right|$, clearly only $\delta$ is determined by $\varepsilon_{0}$. Therefore, $\forall \varepsilon_{0}>0, \exists \delta\left(\varepsilon_{0}\right)$, such that $\rho\left(\pi(t, p), \Gamma_{* *}\right)<\varepsilon_{0}$ for $\forall p \in U\left(p_{* *}, \delta\right)$ when $t \geq t_{0}$. Thus, $\Gamma_{* *}$ is orbitally stable. Then, $\Gamma_{* *}$ is orbitally asymptotically stable.

This completes the proof.

Note 1. Theorem 16 means that an order-1 periodic solution moves toward the inside of the order-1 periodic solution $\Gamma_{*}$ along the phase set and the impulsive set when $q$ changes appropriately from $q=q_{1}$ to $q<q_{1}$.

Similar to the method used for the proof of Theorem 16, the following theorem exists (the proof is omitted).

Theorem 17. In system (1), supposing that there exists an order-1 periodic solution when $q=q_{2}, \tau \geq 0$, and $y_{R}<y_{H}$, then there exists an order-1 periodic solution $\Gamma_{* * *}$ inside the order-1 periodic solution $\Gamma_{*}$ when $q=q_{1}+\vartheta$, where $\vartheta>0$ is sufficiently small. In addition, if $\Gamma_{*}$ is orbitally asymptotically stable, then $\Gamma_{* * *}$ is orbitally stable at least.

Note 2. Theorem 17 means that an order-1 periodic solution moves toward the inside of the order-1 periodic solution $\Gamma_{*}$ along the phase set and the impulsive set when $q$ changes appropriately from $q=q_{1}$ to $q>q_{1}$.

\section{Numerical Simulation and Analysis}

In this section, numerical simulations are presented that verify the correctness of the theoretical results. In particular, using these results, we analyze the feasibility of the artificial and biological methods, and the role of the controlling factor is also discussed. The zooplankton population in the original model was replaced with a fish population in our model [2], but the parameter values used were still those from the previous study [2]; that is, $r=0.3 /$ day, $K=108 \mu \mathrm{g} / \mathrm{L}$, $a=0.7 /$ day, $b=32.49 \mu \mathrm{g} / \mathrm{L}, \varepsilon=0.05$, and $m=0.012 /$ day. Therefore, $E_{2}=\left(x^{*}, y^{*}\right) \approx(4.1172,4.9503)$, which is a stable focus. Thus, $h=3.8 \mu \mathrm{g} / \mathrm{L}$ was assumed.

4.1. Numerical Simulation. In this subsection, assuming that $p=0.8$, the semitrivial solution of system (1) is described as follows:

$$
\begin{gathered}
x(t)=\frac{82.08 \exp (0.3 t-1.638 n)}{107.24+0.76 \exp (0.3 t-1.638 n)} \\
y(t)=0,
\end{gathered}
$$




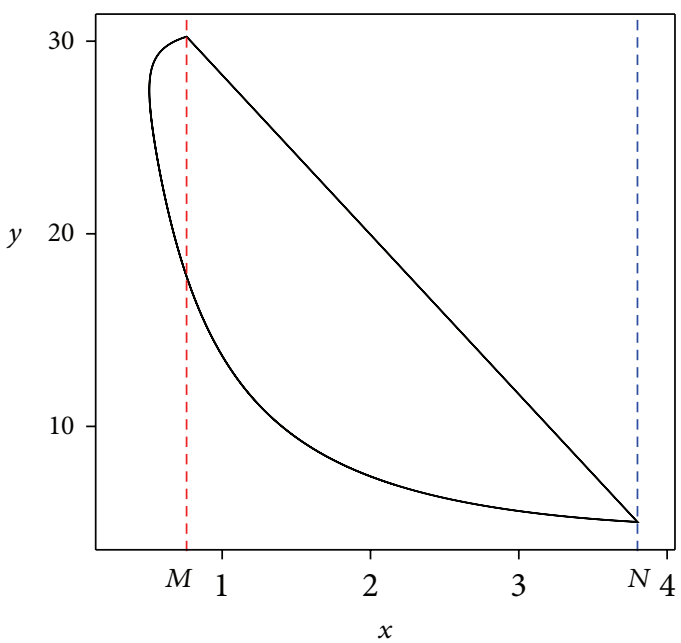

(a)

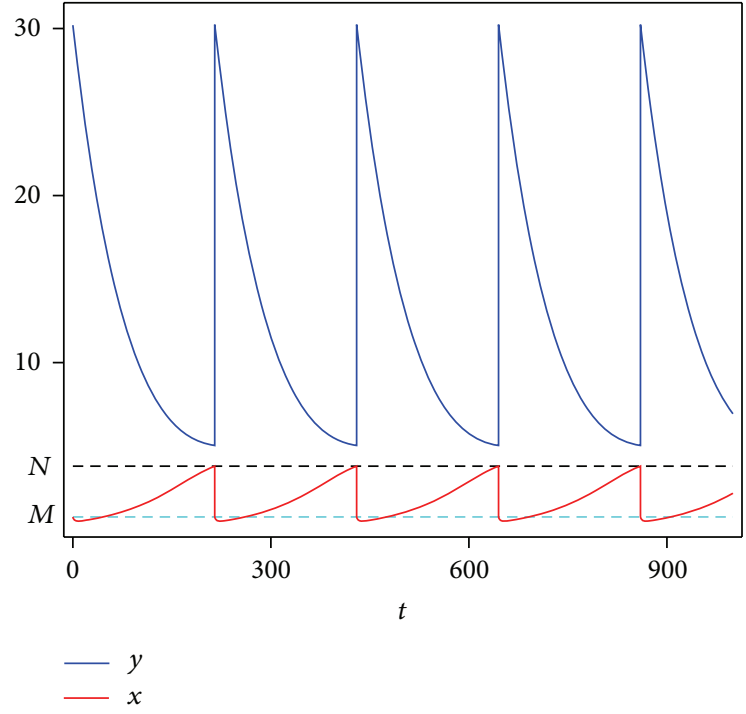

(b)

Figure 5: (a) An order-1 periodic solution to system (1). (b) The time-series that corresponds to the order-1 periodic solution, where the blue curve denotes population $y$ and the red curve denotes population $x(q=1, \tau=0, p=0.8)$.

where $t \in(5.46 n, 5.46(n+1)], n \in N$, and the period $T \approx 5.46$ day. According to Theorem 7, the semitrivial solution is orbitally asymptotically stable when $0<q<$ 0.0456. From Theorem 14, a transcritical bifurcation occurs when $q=q_{*} \approx 0.0456$; that is, the semitrivial solution is unstable when $q \geq q_{*}$. Thus, an order-1 periodic solution appears. Figure 4 verifies the correctness of these results. In Figure 4(a), the trajectory is far from the semitrivial solution (red curve) when $q=0.1>q_{*}$, while the trajectory converges towards the semitrivial solution (blue curve) when $q=0.01<q_{*}$, where their initial points are the same. In Figure 4(b), we can see that a transcritical bifurcation occurs when $q \approx 0.0456$. When $0<q<0.0456$, the semitrivial solution is stable. If $q>0.0456$, however, an order-1 periodic solution emerges that coexists with the semitrivial solution. However, the semitrivial solution is unstable, whereas the order-1 periodic solution is stable.

According to Theorems 9 and 12, there should be an order-1 periodic solution to system (1) when $\tau>0$ or $\tau=$ 0 and $q>0.0456$. This is shown in Figures 5 and 6(a). Figure 5 shows an order-1 periodic solution when $q=1$ and $\tau=0$. Figure 5(a) is the phase diagram and Figure 5(b) is the time series plot with respect to Figure 5(a). In Figure 5, we can see that population $y$ reaches its minimum value when population $x$ reaches its maximum value. However, population $x$ does not reach its minimum value when population $y$ reaches its maximum value. In Figure 5(b), when population $y$ reaches its maximum value, population $x$ reaches the value represented by the cyan line, which is clearly not the minimum value.

Figure 6(a) shows that there exists an order- 1 periodic solution to system (1) when $\tau=0.8$ and $q=1$. In Figure 6(a), the black trajectory is an order-1 periodic solution. The yellow curve is the trajectory of system (1). Clearly, these trajectories are attracted by the order-1 periodic solution. The trajectories of the differential equation do not meet each other in system (1), so Figure 6(a) implies that the order-1 periodic solution is orbitally stable. Thus, the proof of Theorem 16, where the order-1 periodic solution is orbitally asymptotic stable if a trajectory of system (1) is attracted by an order-1 periodic solution, is correct. From $f(h)(1+q)+\tau=f((1-p) h)$, we can obtain $q \approx 2.625$ when $\tau=0$. Thus, according to Theorem 14, there exists an attractor in system (1) when $q<2.625$. Figure 6 (b) shows an attractor of system (1), where $q=0.005$.

From Theorems 16 and 17, there is an order-1 periodic solution when $q=q_{1}$, while an order-1 periodic solution splits away from the order-1 periodic solution when $q>q_{1}$. In Figure $7(\mathrm{a})$, when $q_{1}=10$, the ordinate of the order-1 periodic $y_{R} \approx 55>18.5 \approx y_{H}$. Thus, according to Theorem 16, there exists a unique order-1 periodic solution inside the order-1 periodic solution when $q=q_{1}-\vartheta$. We set $\vartheta$ as equal to 2 and 5, respectively. The results are shown in Figure 7(a). From the expression $q=q_{1}-\vartheta$ and Theorem 16, an order1 periodic solution moves toward the inside of the order-1 periodic solution along the phase set and the impulsive set when $q$ changes appropriately from $q=q_{1}$ to $q<q_{1}$. This is shown clearly in Figure 7(a). Figure 7(b) shows the validity of Theorem 17, where $y_{R} \approx 9<18.5 \approx y_{H}, \tau=0.1$.

These numerical simulations prove that the theoretical result is correct. Next, we analyze the feasibility of an approach including artificial and biological methods using the theoretical results and the numerical simulation.

4.2. Numerical Analysis. The question we need to answer is whether artificial and biological methods can control phytoplankton blooms. We also need to know which method is better. Figure 8 illustrates the difference. In Figure 8(a), 


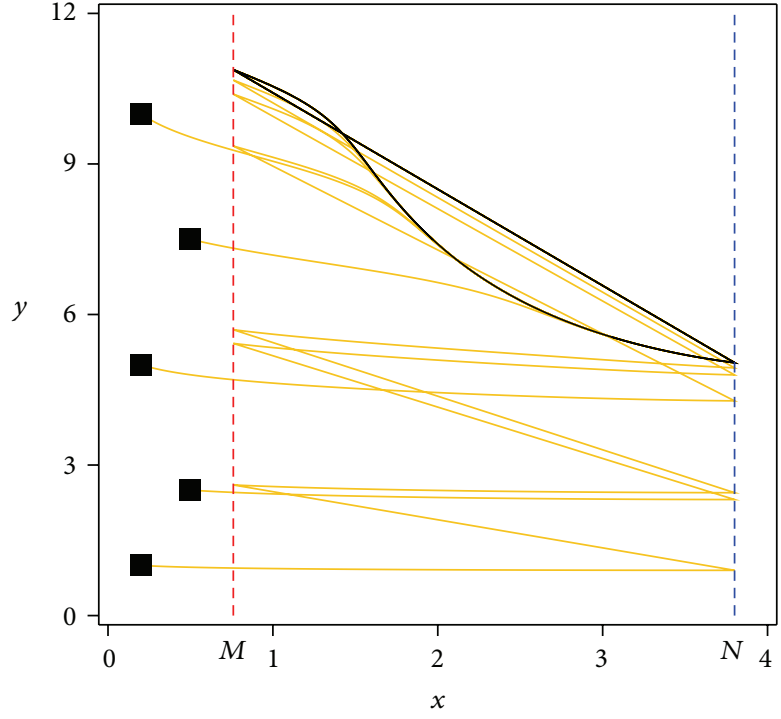

(a)

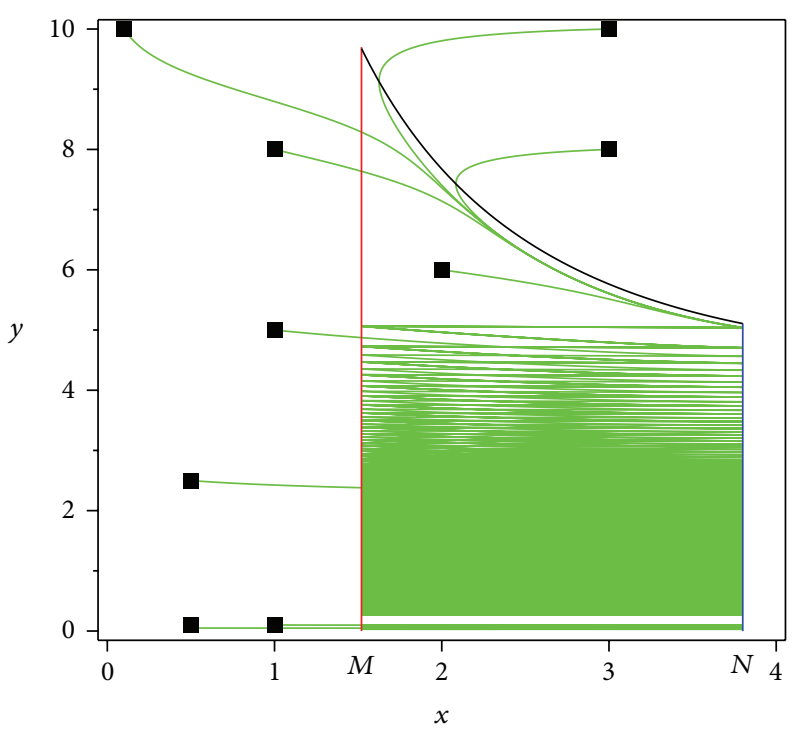

(b)

Figure 6: (a) The black curve denotes an order-1 periodic solution to system (1), where the yellow curve denotes the trajectory in system (1) and the black symbol denotes the initial point, $\tau=0.8, q=1, p=0.8$. (b) The region that comprises the red line, blue line, and black curve is an attractor in system (1), where the green curve denotes the trajectory of system (1) and the black symbol denotes the initial point, $q=0.005$, $\tau=0, p=0.8$.

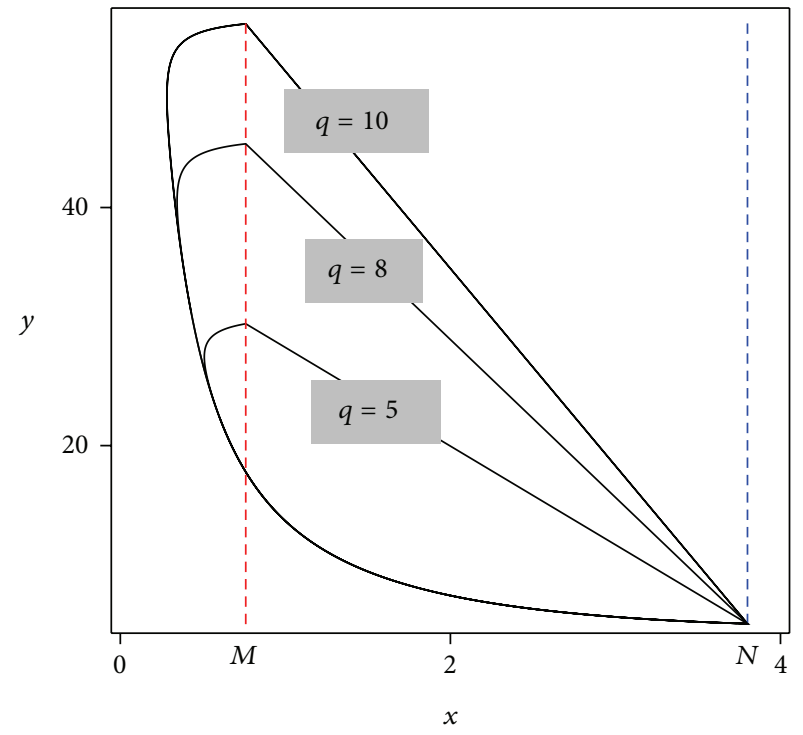

(a)

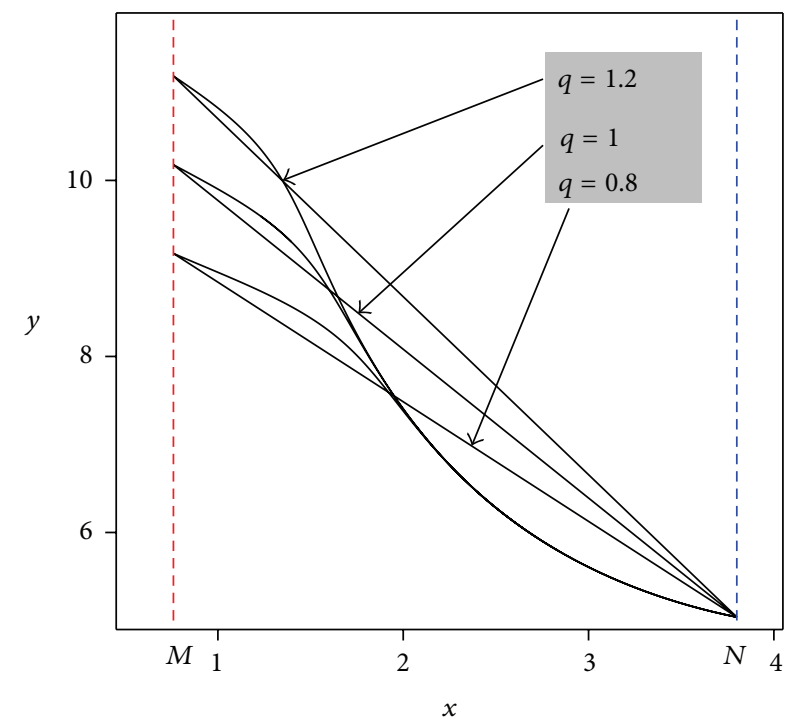

(b)

FIgURE 7: Bifurcation of an order-1 periodic solution. (a) $y_{R} \approx 55>18.5 \approx y_{H}, p=0.8, \tau=0$; (b) $y_{R} \approx 9<18.5 \approx y_{H}, p=0.8, \tau=0.1$.

only the biological method is considered, where the red curve corresponds to the right vertical axis, while the blue curve and the green curve correspond to the left vertical axis, where the initial densities of phytoplankton and fish are $3 \mu \mathrm{g} / \mathrm{L}$ and $5 \mu \mathrm{g} / \mathrm{L}$, respectively. In Figure 8(a), when $q$ equals 2 and 5, respectively, the biological method alone cannot control the growth of phytoplankton. When the density of phytoplankton is below the critical value $h$, phytoplankton blooms occur. When $q=40$, the growth of phytoplankton is controlled.
However, the density of fish released is 40 times the existing density of fish, but we do not know whether all of the released fish can be accommodated by the current environment. In addition, when $q=40$, we find that blooms occur from the 200th day in Figure 8(a).

In Figure 8(b), only the artificial method is considered and the initial densities of the phytoplankton and fish are $3 \mu \mathrm{g} / \mathrm{L}$ and $5 \mu \mathrm{g} / \mathrm{L}$, respectively. Clearly, the density of phytoplankton remains below the critical value $h$ because of 


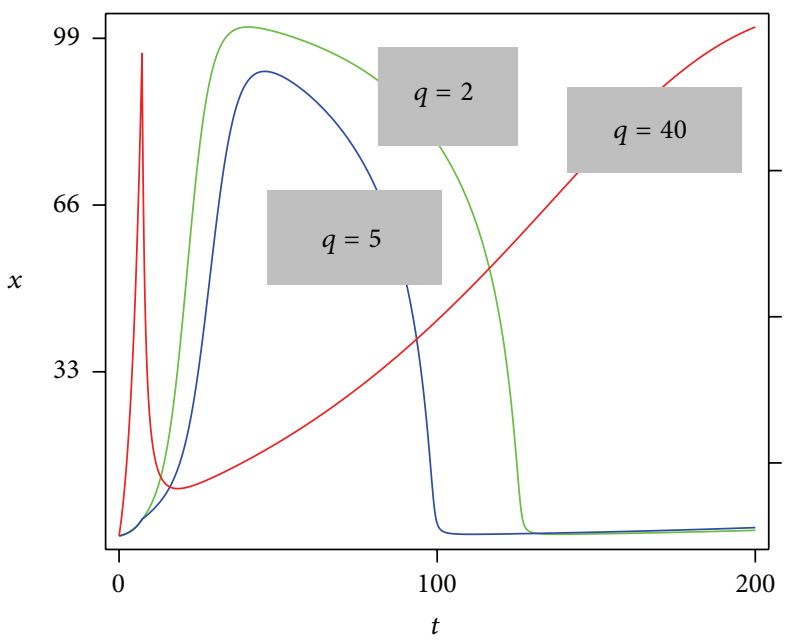

(a)

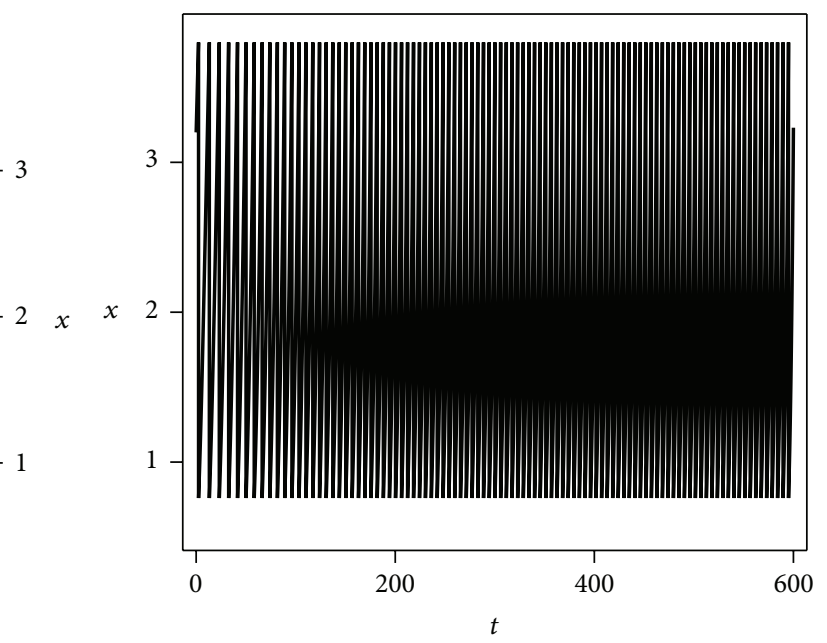

(b)

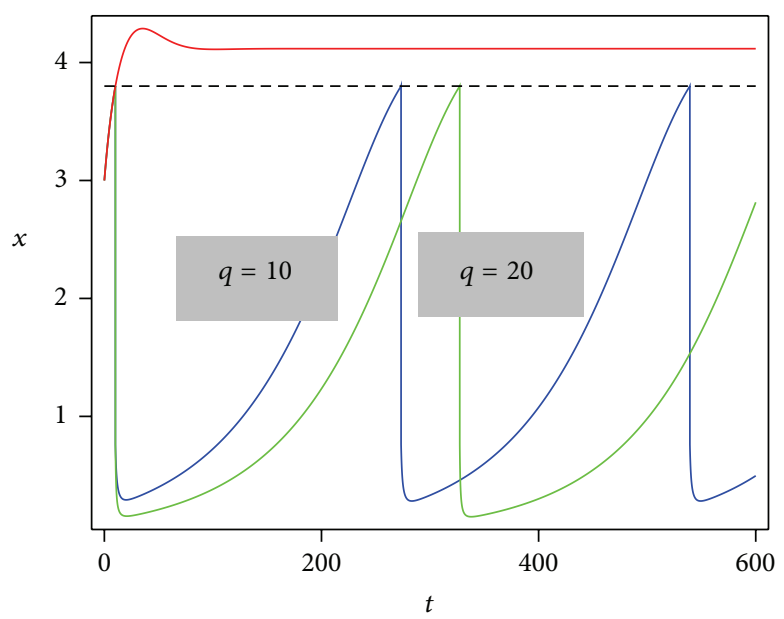

(c)

FIGURE 8: Time-series of system (1). (a) Only with the biological method, $p=0, \tau=0$; (b) only with the artificial method, $q=0, \tau=0$; (c) with the biological and artificial methods, $p=0.8, \tau=0$, where the red curve denotes the case without control.

artificial control. However, too many phytoplankton blooms occur within 600 days. Figure 8(b) shows that too much artificial control is required when only the artificial method is used. Indeed, if the density of fish is much lower and certain conditions are satisfied, such as the nutrient levels and sufficient illumination, phytoplankton blooms occur again after several days of artificial control. These phenomena have been observed in reality.

In Figure 8(c), both the artificial and biological methods are considered, where the initial densities of phytoplankton and fish are $3 \mu \mathrm{g} / \mathrm{L}$ and $5 \mu \mathrm{g} / \mathrm{L}$, respectively, and $p=0.8$. The red curve represents the change in the phytoplankton density without artificial and biological control. From Figure 8(c), it is clear that the density of phytoplankton remains above the critical value $h$ several times during the 600-day period when the artificial and biological methods are not implemented. Figure 8(c) shows that a phytoplankton bloom occurs three times when $q=10$ and twice in about 500 days. When $q=20$, there are two phytoplankton blooms, with one in about 500 days. Compared with Figures 8(a) and 8(b), it is clear that the results shown in Figure 8(c) are much better. Thus, both the artificial and biological methods should be used.

According to Theorem 7, when $0<q<q_{*}$, the semitrivial periodic solution is orbitally asymptotically stable. From Figure 4(a), however, we find that the time interval of phytoplankton blooms is much shorter when $0<q<q_{*}$. This suggests that too much artificial control is needed. Thus, $q$ should be larger than $q_{*}$ and the value of $q_{*}$ with respect to $p$ and $h$ is given in Figure 9(a). In addition, from Theorem 14, when $0<q<\left((K-(1-p) h)\left(b+((1-p) h)^{2}\right) /(1-\right.$ $\left.p)(K-h)\left(b+h^{2}\right)\right)-\left(a K h \tau / r(K-h)\left(b+h^{2}\right)\right)-1 \stackrel{\text { define }}{\longrightarrow} q_{+}$, there exists an attractor in system (1). In Figure 6(b), when artificial and biological controls are implemented, although the phytoplankton blooms are contained, the density of phytoplankton still increases, because the released fish cannot control the growth of phytoplankton. In Figure 7(a), the released fish controlled the growth of phytoplankton only when the value of $q$ is beyond a certain value. Indeed, 


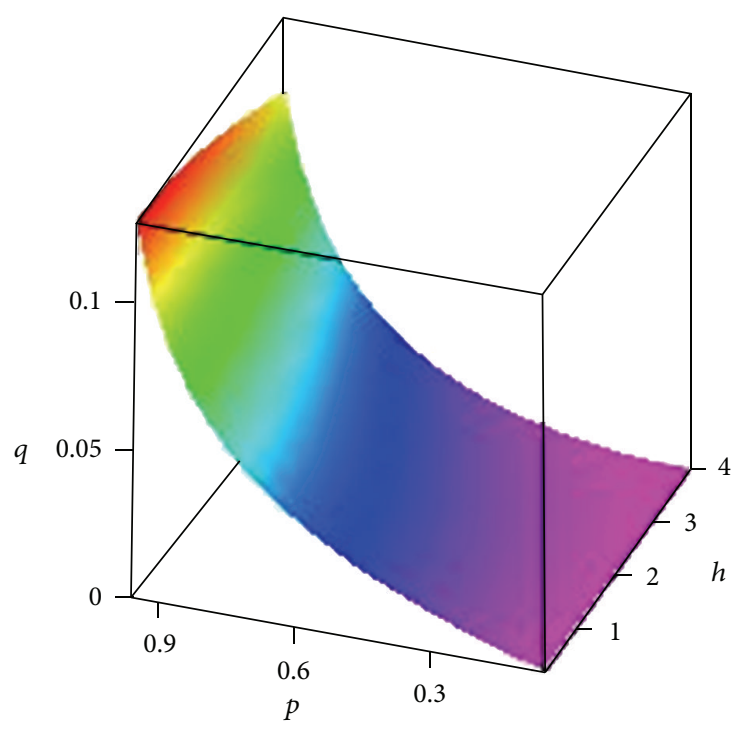

(a)

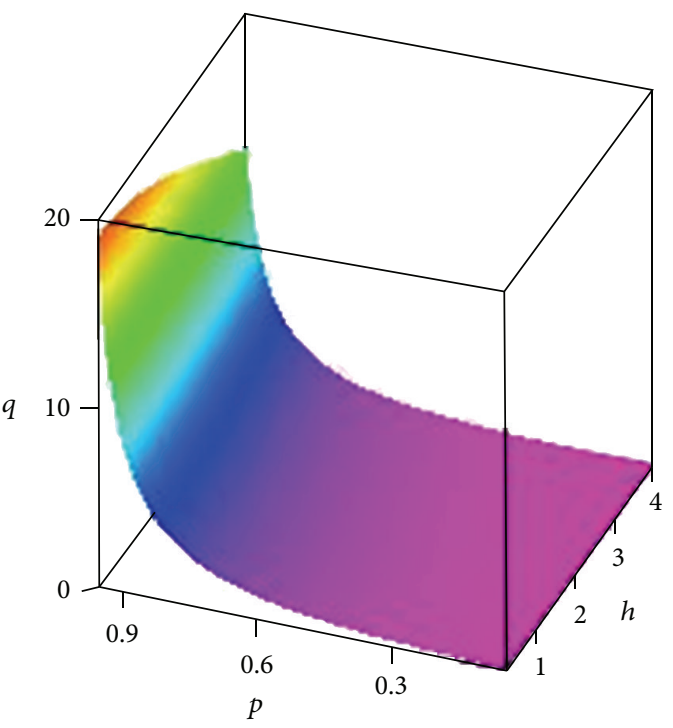

(b)

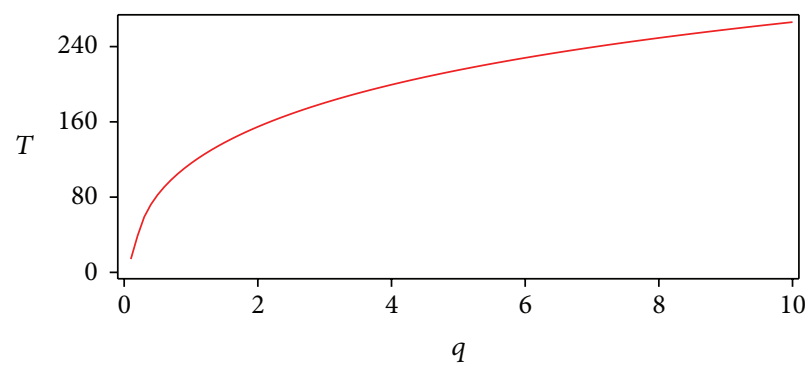

(c)

FIGURE 9: (a) Relationship between $q_{*}$ with respect to $p$ and $h$ from Theorem $7, \tau=0$. (b) The relationship between $q_{+}$with respect to $p$ and $h$ from Theorem 14, $\tau=0$. (c) The relationship of the time interval between two controls with respect to $q, p=0.8, h=3.8, \tau=0$.

from Theorem 14, we know that the value is $q_{+}$. Figure 9(b) shows the relationships among the value $q_{+}, p$, and $h$. The released fish always controlled the growth of phytoplankton within a certain period of time when $q>q_{+}$. We set the values of $h, \tau$, and $p$ to $3.8 \mu \mathrm{g} / \mathrm{L}, 0 \mu \mathrm{g} / \mathrm{L}$, and $0.8 \mu \mathrm{g} / \mathrm{L}$, respectively. The relationship between $q$ and the average time interval $T$, which is the time between two implemented controls, is shown in Figure 9(c) for 1000 days, where the initial densities of phytoplankton and fish are $3 \mu \mathrm{g} / \mathrm{L}$ and 5 $\mu \mathrm{g} / \mathrm{L}$, respectively. Figure 9(c) shows that the time interval $T$ also increases when $q$ increases; that is, the frequency of phytoplankton blooms decreases with increased biological control. Indeed, according to Theorems 16 and 17, the order1 periodic solution moves the phase set and the impulse moves from the bottom to top with the increase in $q$. If the order-1 periodic solution is stable, the trajectory will converge toward the order-1 periodic solution. It is clear that the time required to reach $x=h$ increases, which is exactly what we hoped. Thus, phytoplankton blooms can be controlled using artificial and biological methods together. Therefore, we should replace the artificial method with biological methods as much as possible. This is feasible according to a previous analysis.

\section{Conclusion and Discussion}

In this study, we developed a phytoplankton-fish model with artificial and biological control, which we studied analytically and numerically. To investigate the feasibility of artificial and biological methods, theoretical mathematical results were required. Thus, we studied the existence and stability of a semitrivial periodic solution and an order-1 periodic solution of system (1) initially, and we proved that a positive periodic solution emerged from the semi-trivial periodic solution via transcritical bifurcation using bifurcation theory. In addition, we discussed the bifurcation of an order-1 periodic solution. We proved that an order-1 periodic solution emerged from the existing order-1 periodic solution. These results are very useful when studying the biological and artificial control of phytoplankton blooms. We also described several numerical simulations, which verified the theoretical results.

Based on previous theoretical and numerical results, we showed that phytoplankton blooms can be controlled using artificial and biological methods. In particular, we demonstrated the feasibility of a method where as many fishes as possible are released and as little phytoplankton is removed as possible; that is, the artificial method should be replaced 
by biological methods as much as possible. However, the results were not very good when we only used a biological method. Likewise, if only the artificial method was used, too much artificial control was required. Thus, our study demonstrated that artificial and biological methods should be used, that is, integrated management. Furthermore, we suggest that fish (or zooplankton) should be released when the density of phytoplankton reaches a critical biological value. Moreover, when the density of phytoplankton reaches the bloom's critical value, phytoplankton should be removed using the artificial method. The results may be better using this approach. The corresponding theoretical results were obtained using the method proposed in the present study.

Although our model is simple and it can only abstract real world phenomena, the model reproduced many real-world features. First, when only artificial method is considered in our model, the density of phytoplankton can be restrained. In reality, artificial method is the main method controlling phytoplankton bloom at present, so the method is effective. Second, when only biological method is considered in our model, the density of phytoplankton cannot be below critical value right now. In reality, when phytoplankton bloom occurs, biological method is hardly applied. Third, in recent years, people control phytoplankton bloom occurs using fish-farming. When phytoplankton bloom occurs, people remove the phytoplankton using chemical reagent. The result is better. While our model shows that adopting biological method and artificial method at the same is much better. Furthermore, our model predicts that the intensity of biological method and artificial method is beyond a certain level; then, the phytoplankton bloom can be controlled. Based on the analysis of our model, we can obtain many results that agreed with reality. These results are expected to be useful for studying algae in Zeya Reservoir and other ecosystems.

\section{Conflict of Interests}

The authors declare that they have no conflict of interests regarding the publication of this paper.

\section{Acknowledgments}

This work was supported by the National Natural Science Foundation of China (Grant no. 31170338), by the Key Program of Zhejiang Provincial Natural Science Foundation of China (Grant no. LZ12C03001), and by the National Key Basic Research Program of China (973 Program, Grant no. 2012CB426510).

\section{References}

[1] R. Reigada, R. M. Hillary, M. A. Bees, J. M. Sancho, and F. Sagués, "Plankton blooms induced by turbulent flows," Proceedings of the Royal Society B, vol. 270, no. 1517, pp. 875-880, 2003.

[2] J. E. Truscott and J. Brindley, "Ocean plankton populations as excitable media," Bulletin of Mathematical Biology, vol. 56, no. 5, pp. 981-998, 1994.
[3] M. Sandulescu, C. López, and U. Feudel, "Plankton blooms in vortices: the role of biological and hydrodynamic timescales," Nonlinear Processes in Geophysics, vol. 14, no. 4, pp. 443-454, 2007.

[4] A. Huppert, B. Blasius, R. Olinky, and L. Stone, "A model for seasonal phytoplankton blooms," Journal of Theoretical Biology, vol. 236, no. 3, pp. 276-290, 2005.

[5] P. Almeida Machado, "Dinoflagellate blooms on the Brazilian South Atlantic coast," in Toxic Dinoflagellate Blooms, Taylor and Seliger, Ed., p. 29, Elsevier, Amsterdam, The Netherlands, 1978.

[6] P. Wu, Z. M. Yu, G. P. Yang, and X. X. Song, "Remediation from harmful algae bloom with organo-clay processed surfactant," Oceanologia et Limnologia Sinica, vol. 37, no. 6, pp. 511-516, 2006.

[7] J. K. Liu and P. Xie, "Unraveling the enigma of the disappearance of water bloom from the east lake (Lake Donghu) of Wuhan," Resources and Environment in the Yangtze Basin, vol. 8, no. 3, pp. 312-319, 1999.

[8] F. L. R. M. Starling, "Control of eutrophication by silver carp (Hypophthalmichthys molitrix) in the tropical Paranoá Reservoir (Brasília, Brazil): a mesocosm experiment," Hydrobiologia, vol. 257, no. 3, pp. 143-152, 1993.

[9] A. D. Carruthers et al., Effect of Silver Carp on Blue-Green Algal Blooms in Lake Orakai, vol. 68 of Fisheries Environmental Report, Ministry of Agriculture and Fisheries, Wellington, New Zealand, 1986.

[10] H. Leventer and B. Teltsch, "The contribution of silver carp (Hypophthalmichthys molitrix) to the biological control of Netofa reservoirs," Hydrobiologia, vol. 191, no. 1, pp. 47-55, 1990.

[11] T. Miura, "The effects of planktivorous fishes on the plankton community in a eutrophic lake," Hydrobiologia, vol. 200-201, no. 1, pp. 567-579, 1990.

[12] V. Lakshmikantham, D. D. Bainov, and P. S. Simeonov, Theory of Impulsive Differential Equations, vol. 6, World Scientific, Singapore, 1989.

[13] D. D. Bainov and P. S. Simeonov, Impulsive Differential Equations: Asymptotic Properties of the Solutions, World Scientific, Singapore, 1993.

[14] D. D. Bainov and V. C. Covachev, Impulsive Differential Equations with a Small Parameter, vol. 24, World Scientific, Singapore, 1994.

[15] C. J. Dai, M. Zhao, and L. S. Chen, "Complex dynamic behavior of three-species ecological model with impulse perturbations and seasonal disturbances," Mathematics and Computers in Simulation, vol. 84, pp. 83-97, 2012.

[16] L. S. Chen, "Pest control and geometric theory of semidynamical systems," Journal of Beihua University, vol. 12, pp. 1-9, 2011.

[17] C. J. Dai, M. Zhao, and L. S. Chen, "Dynamic complexity of an Ivlev-type prey-predator system with impulsive state feedback control," Journal of Applied Mathematics, vol. 2012, Article ID 534276, 17 pages, 2012.

[18] W. B. Wang, J. H. Shen, and J. J. Nieto, "Permanence and periodic solution of predator-prey system with holling type functional response and impulses," Discrete Dynamics in Nature and Society, vol. 2007, Article ID 81756, 15 pages, 2007.

[19] C. J. Dai, M. Zhao, and L. S. Chen, "Homoclinic bifurcation in semi-continuous dynamic systems," International Journal of Biomathematics, vol. 5, no. 6, pp. 1-19, 2012.

[20] L. N. Qian, Q. S. Lu, Q. Meng, and Z. Feng, "Dynamical behaviors of a prey-predator system with impulsive control," 
Journal of Mathematical Analysis and Applications, vol. 363, no. 1, pp. 345-356, 2010.

[21] H. Baek, "Extinction and permanence of a three-species LotkaVolterra system with impulsive control strategies," Discrete Dynamics in Nature and Society, vol. 2008, Article ID 752403, 18 pages, 2008.

[22] C. J. Dai and M. Zhao, "Mathematical and dynamic analysis of a prey-predator model in the presence of alternative prey with impulsive state feedback control," Discrete Dynamics in Nature and Society, vol. 2012, Article ID 724014, 19 pages, 2012.

[23] D. L. deAngelis, S. M. Bartell, and A. L. Brenkert, "Effects of nutrient recycling and food-chain length on resilience," The American Naturalist, vol. 134, no. 5, pp. 778-805, 1989.

[24] A. M. Edwards, "Adding detritus to a nutrient-phytoplanktonzooplankton model: a dynamical-systems approach," Journal of Plankton Research, vol. 23, no. 4, pp. 389-413, 2001.

[25] T. Wyatt and J. Horwood, "Model which generates red tides," Nature, vol. 244, no. 5413, pp. 238-240, 1973.

[26] S. Uye, "Impact of copepod grazing on the red tide flagellate Chattonella antiqua," Marine Biology, vol. 92, no. 1, pp. 35-43, 1986.

[27] S. A. Levin and L. A. Segel, "Hypothesis for origin of planktonic patchiness," Nature, vol. 259, no. 5545, p. 659, 1976.

[28] G. Pan, M. M. Zhang, H. Yan, H. Zou, and H. Chen, "Kinetics and mechanism of removing Microcystis aeruginosa using clay flocculation," Environmental Science, vol. 24, no. 5, pp. 1-10, 2003.

[29] H. Zou, G. Pan, and H. Chen, "Flocculation and removal of water bloom cells Microcystis aeruginosa by Chitosan-Modified Clays," Environmental Science, vol. 25, no. 6, pp. 40-43, 2004.

[30] P. S. Simeonov and D. D. Bainov, "Orbital stability of periodic solutions of autonomous systems with impulse effect," International Journal of Systems Science, vol. 19, no. 12, pp. 2561-2585, 1988.

[31] S. N. Rasband, Chaotic Dynamics of Nonlinear Systems, John Wiley \& Sons, New York, NY, USA, 1990. 


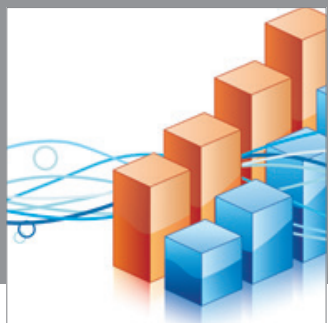

Advances in

Operations Research

mansans

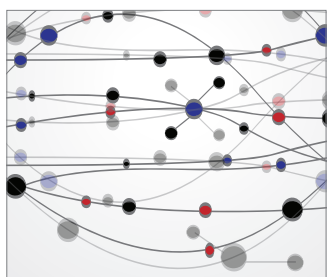

The Scientific World Journal
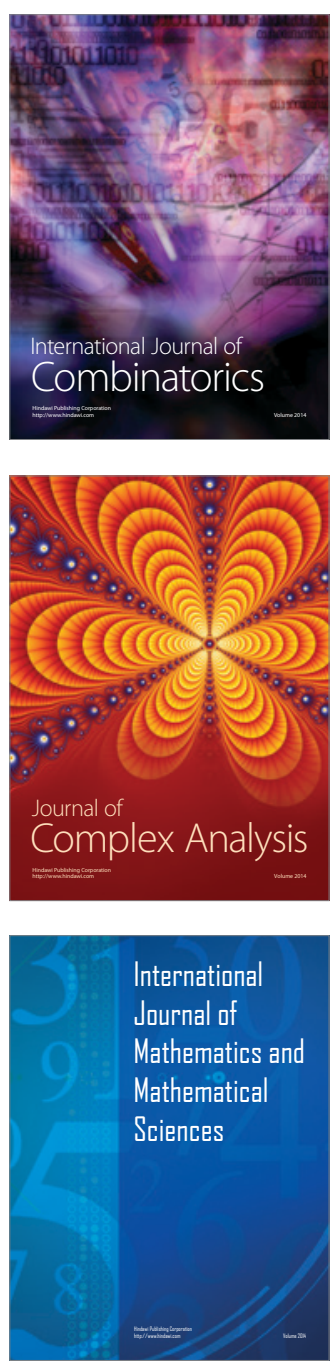
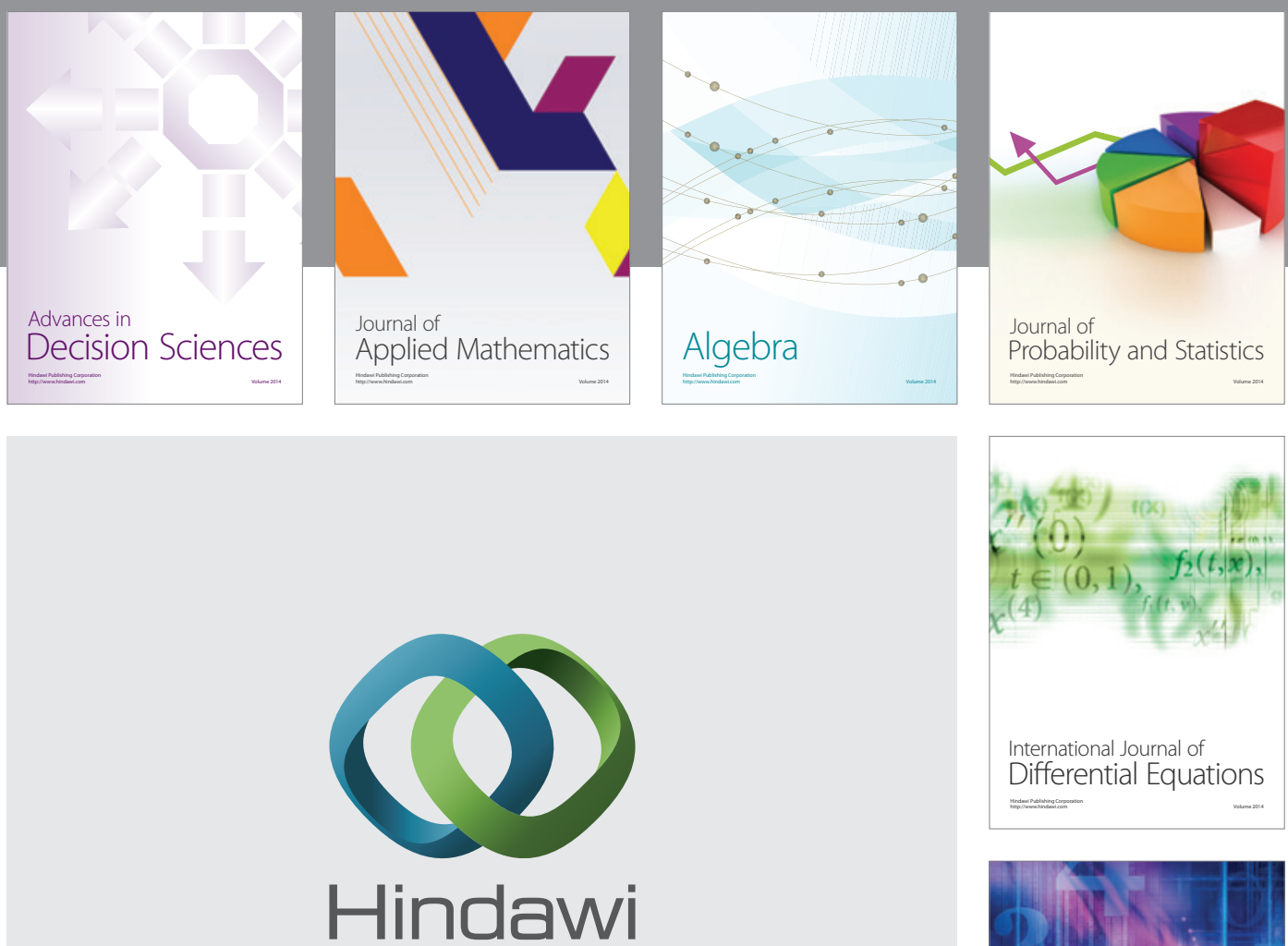

Submit your manuscripts at http://www.hindawi.com
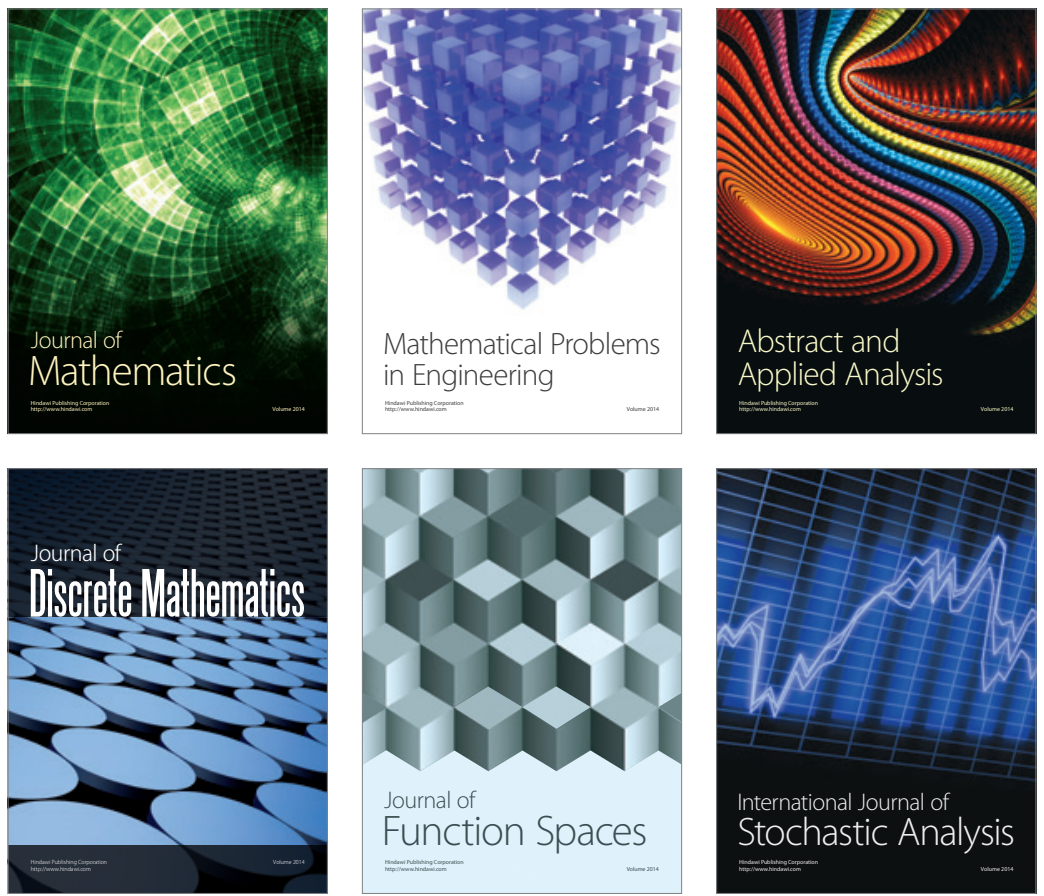

Journal of

Function Spaces

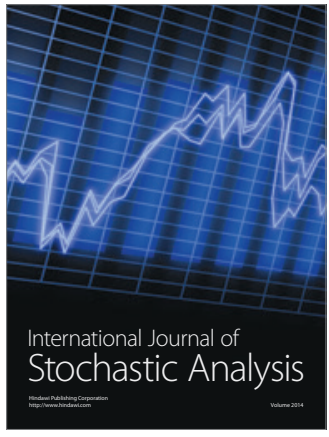

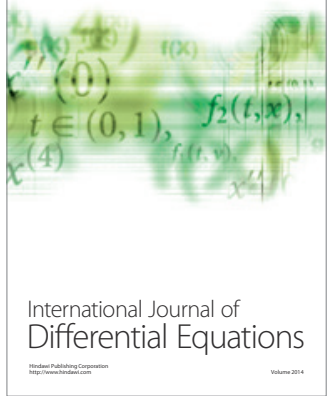
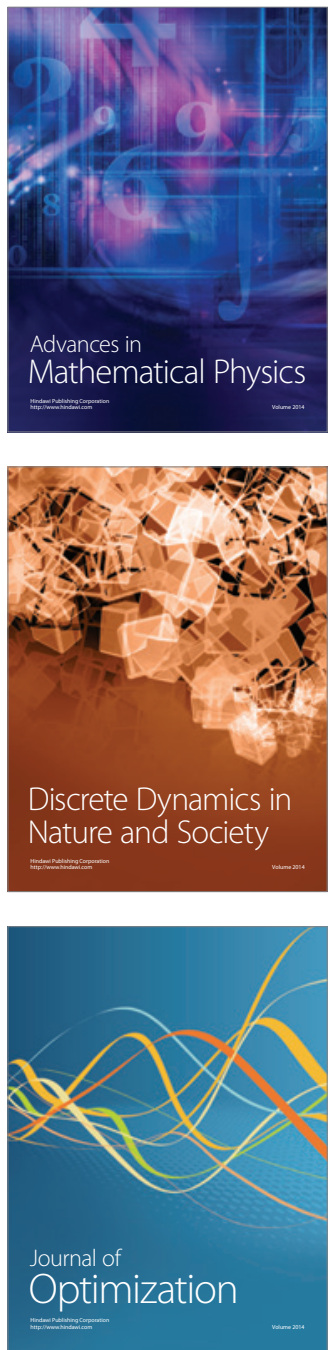